\title{
The All-Source Green's Function of Linear Shallow Water Dynamic System: Its Numerical Constructions and Applications to Tsunami Problems
}

\author{
Zhigang $\mathrm{Xu}$ \\ Modelling and Operational Oceanography, Canadian Hydrographic Service, Maurice \\ Lamontagne Institute, Fisheries and Oceans
}

Canada

\section{Introduction}

Propagation of tsunamis in deep water (>50 m, Shuto, 1991) obeys linear dynamics. For a linear dynamics system, any response of the system to arbitrary external forcing can be expressed as a linear combination of the Green's functions. The Green's functions can be pre-calculated for fixed physical parameters and geometry of the system, whereas the precalculation implies a great time saving, which is essential when a catastrophe occurs and an immediate solution is required.

A Green's function has traditionally been calculated as a domain wise solution to a unitimpulse acting at a grid point. Such a Green's function may be referred as a single-source Green's function (SSGF). However a tsunami is rarely triggered at a single source point. A reasonable extension is therefore to pre-calculate a set of such SSGFs corresponding to a group of source points in a pre-assumed tsunami source region. Nevertheless when a future tsunami happens outside of the pre-assumed source region, the pre-calculated SSGFs will not be helpful.

$\mathrm{Xu}$ (2007) proposed a new type of Green's function, an all-source Green's function (ASGF). An ASGF focuses on a receiver point, regarding all the model grid points as potential sources. The computational cost for an ASGF is the same as that for an SSGF. However an ASGF provides thorough preparedness for a point of interest (POI) against all possible sources. Tsunamis from different sources may arrive at a POI at different times and may have different maximum wave amplitudes within certain period. Xu showed that with an ASGF one could make an arrival time map and a map of relative maximum amplitudes for a POI. $\mathrm{Xu}$ further demonstrated that with the ASGFs, one could also establish a real-time simulation system, which would give tsunami arrival time series at one or more POIs immediately.

This chapter will describe in details the ASGF in terms of its numerical constructions and its applications to tsunami problems. It will first present the governing equations and boundary conditions. It will move on to grid auto-generations from a realistic topographic 
dataset. It will then show how to build the global operators, with which the shallow water equations can be recast as a single matrix equation, with all the dynamics and boundary conditions encapsulated. Considerations will be given on how to stabilize the matrix before it can be used iteratively. With all these preparations, the chapter will then show how the ASGF can easily be calculated from the dynamics matrix, as easy as to calculate the traditional SSGF.

Section 7 will discuss applications of the ASGF to tsunami problems in terms of both tsunami preparedness and real-time simulations. The section will show how the ASGFs can be used for making tsunami arrival time maps and tsunami amplitude maps for a POI. A web-based real-time simulation system for the India and Pacific Oceans will also be demonstrated. The simulation system, which can be found at odylab.uqr.ca ${ }^{1}$, allows its users to see the tsunami arrival time series at one or several POIs, upon receiving the source region specified by users on its interactive map.

\section{Linear Shallow Water Equations (LSWE) and boundary conditions}

\subsection{Continuous form of LSWE}

The governing equations consist of

$$
\begin{aligned}
& \frac{\partial \eta}{\partial \mathrm{t}}=-\frac{1}{\mathrm{R} \cos \varphi}\left(\frac{\partial \mathrm{U}}{\partial \lambda}+\frac{\partial \mathrm{V} \cos \phi}{\partial \varphi}\right) \\
& \frac{\partial \mathrm{U}}{\partial \mathrm{t}}=-\frac{\mathrm{gh}}{\mathrm{R} \cos \varphi} \frac{\partial \eta}{\partial \lambda}+\mathrm{fV}-\kappa \frac{\mathrm{U}}{\mathrm{h}} \\
& \frac{\partial \mathrm{V}}{\partial \mathrm{t}}=-\frac{\mathrm{gh}}{\mathrm{R}} \frac{\partial \eta}{\partial \varphi}-\mathrm{fU}-\kappa \frac{\mathrm{V}}{\mathrm{h}}
\end{aligned}
$$

The meaning of the notation is listed below:

\begin{tabular}{|l|l|}
\hline$\lambda, \varphi, \mathrm{t}$ & Longitude, latitude, and time variables respectively. \\
\hline $\mathrm{\eta}, \mathrm{U}, \mathrm{V}$, & $\begin{array}{l}\text { Sea surface elevation, and components of total stream in longitudinal and } \\
\text { latitudinal directions. }\end{array}$ \\
\hline $\mathrm{f}, \mathrm{g}, \mathrm{R}$, & Coriolis parameter, gravity acceleration, and the Earth's mean radius. \\
\hline $\mathrm{h}, \mathrm{K}$ & Water depth and bottom frictional coefficients. \\
\hline
\end{tabular}

The components of total stream are defined as the following depth integrals

$$
\mathrm{U}=\quad \int_{-\mathrm{h}}^{0} \mathrm{udz}, \quad \mathrm{V}=\int_{-\mathrm{h}}^{0} \mathrm{vdz},
$$

where $\mathrm{u}$ and $\mathrm{v}$ are eastward and northward velocity components respectively. The total stream may also be referred as the mass transport.

\subsection{Boundary conditions}

The boundary conditions consist of zero flows normal to the coasts,

\footnotetext{
${ }^{1}$ If you find this becomes inaccessible, please contact me at zhigang.xu@dfo-mpo.gc.ca or at zhigang_xu_98@yahoo.com.
} 


$$
\begin{array}{lc}
\mathrm{U}=0 & \text { at the west and east coasts } \\
\mathrm{V}=0 & \text { at the south and north coasts }
\end{array}
$$

and the Sommerfeld radiation conditions at open water boundaries,

$$
\frac{\partial \mathrm{p}}{\partial \mathrm{t}}+\mathrm{c} \frac{\partial \mathrm{p}}{\partial \mathrm{n}}=0 \quad \text { at open water boundaries }
$$

where $\mathrm{p}$ may stand for $\mathrm{U}, \mathrm{V}$ or $\eta, \mathrm{c}$ is a phase speed, and $\partial n$ means an infinitesimal line segment along the directions outward and normal to the open boundaries. Chapman (1985) discussed various forms of the phase speed and their numerical effects on the quality of the model solutions inside of the model domain. For this study, the long gravity wave $c=\sqrt{g h}$ is taken.

\subsection{Frictional coefficient as a function of water depth}

The last terms on RHS of Eqs. (2) and (3) represent the frictional effects of the seabed on the water column above. Their forms imply that the bottom frictional force is linearly proportional to the depth averaged velocities. The coefficient $\mathrm{K}$ reflects the proportionality. Heaps (1969) simply took a constant $\mathrm{k}, 0.0024 \mathrm{~m} / \mathrm{s}$, for his two-dimensional numerical model in the North Sea. It is desirable to have a spatially varying $\mathrm{K}$ since there is less friction in deep water than in shallow water. To derive $\mathrm{K}$ as a function of $\mathrm{h}$, we may go back to a more general frictional parameterization as follows (e.g., Brummelhuis et al, 1993)

$$
\left(\tau_{\mathrm{x}}^{\mathrm{b}}, \tau_{\mathrm{y}}^{\mathrm{b}}\right)=\frac{\mathrm{g} \sqrt{\overline{\mathrm{u}}^{2}+\overline{\mathrm{u}}^{2}}}{\mathcal{C}^{2}}(\overline{\mathrm{u}}, \overline{\mathrm{v}})
$$

where $\mathcal{C}$ is the Chezy's coefficient, whose unit is $\mathrm{m}^{1 / 2} \mathrm{~s}^{-1}$. This is a non-linear form in terms of the velocities, the stresses being a quadric function of the velocity components. $\sqrt{\bar{u}^{2}+\bar{u}^{2}}$ is usually replaced by an estimate of the magnitude of background currents, in order to preserve the linearity of the whole system. Let $|w|$ represents such an estimate. Further the Chezy's coefficient can be linked with water depth as $\mathcal{C}=\frac{1}{\mathcal{N}} \mathrm{h}^{1 / 6}$ where $\mathcal{N}$ represents Manning's roughness. Now Eq.(8) can be transformed as

$$
\left(\tau_{x}^{b}, \tau_{y}^{b}\right)=\kappa(\bar{u}, \bar{v})
$$

with

$$
\kappa=\frac{\mathrm{g} \mathcal{N}^{2}|\mathrm{w}|}{\mathrm{h}^{1 / 3}}
$$

as desired.

The tidal currents are usually taken as the background currents in oceans, whose typical value is of order of $0.1 \mathrm{~m} / \mathrm{s}$. The Manning's roughness in ocean appears to be on a range of 0.01 to 0.4 (Ding et al 2004; Fujima 2001). With $|\mathrm{w}|=0.1 \mathrm{~m} / \mathrm{s}$, if we let $\mathcal{N}$ be 0.1 and $h$ be $65 \mathrm{~m}$ to represent the mean water depth of the North Sea (Heaps, 1971), we can recover $\kappa=0.0024 \mathrm{~m} / \mathrm{s}$ as used by Heaps (1969). If we still use $\mathcal{N}=0.1$ but let $\mathrm{h}=4000 \mathrm{~m}$ as typical water depth in deep 
oceans, we can have $\kappa=6 \times 10^{-4} \mathrm{~m} / \mathrm{s}$. For the India-Pacific model domain, the minimum, mean and max values of $\kappa$ are $4.5 \times 10^{-4} \mathrm{~m} / \mathrm{s}, 7.6 \times 10^{-4} \mathrm{~m} / \mathrm{s}$ and $4.6 \times 10^{-3} \mathrm{~m} / \mathrm{s}$.

\subsection{A matrix equation to be derived for hosting discrete LSWE}

It is necessary to discretize the continuous form of LSWE and boundary conditions in order to be able to solve them numerically. Discretization for such a purpose is a common practice, but what is pursued here is beyond. The goal here is to recast the shallow water equations in the following matrix form:

$$
\left[\begin{array}{l}
\eta \\
U \\
V
\end{array}\right]^{(\tau+1)}=A\left[\begin{array}{l}
\eta \\
U \\
V
\end{array}\right]^{(\tau)}
$$

where $\tau$ is an index of the time step. The coefficient matrix A, encapsulates the model physics and geometry, hence it may also be called the dynamics matrix. It is also called propagation matrix because it propagates the solutions from the current time step to the next. The column vector $[\eta \mathrm{U} \mathrm{V}]^{\mathrm{T}}$ consists of all the model variables defined on the grid points.

There are advantages in having such a matrix form, especially for tsunami problems, as will be shown later on. In order to arrive at Eq.(11), we have to first construct some global operators, which in turn require an organized grid beforehand.

\section{An organized rectangular grid}

A rectangular grid has been the simplest to construct comparing with the other types of grids (e.g. a triangular grid): two sets of straight lines plus a land masking matrix equals a grid. However for assembling the dynamics matrix A, such a simple approach is not good enough, because all the entries into the matrices and vectors must be valid. We have to go above this basic level.

\subsection{Issues encountered in generating grid from a topographic dataset}

The starting point for generating a grid is a topographic dataset. From a topographic dataset to a valid grid, we will encounter some issues and have to address them well before we can have a valid grid.

Shown in Panel (a) of Figure 1 is a mini topographic dataset. The dataset is cell oriented, meaning that the water depths or land heights are defined on the centers of the cells. The cells are outlined by the two sets of thick straight lines. The centers of the cells are intersected by the two sets of thin straight lines and also marked by the blue dots. The gray cells are land cells and white ones are water cells. This mini topographic dataset contains all the issues that have to be addressed during the grid generation:

1. Singletons. A singleton is a single water cell not connected with others. Examples of singletons in Panel (a) are the water cells centered at $(i=14, j=34)$, and the one at $(i=38$, $\mathrm{j}=14$ ). There is no need to retain singletons. Retaining them not only wastes computational resources, but may also be harmful because they may mislead the judgment of minimum of the widths of the water cells. When a singleton lies on higher latitude than all other water cells, the width of that singleton would dictate the minimum value of the horizontal grid spacing, which would in turn cause the time step unnecessarily smaller than it could be without that singleton. 

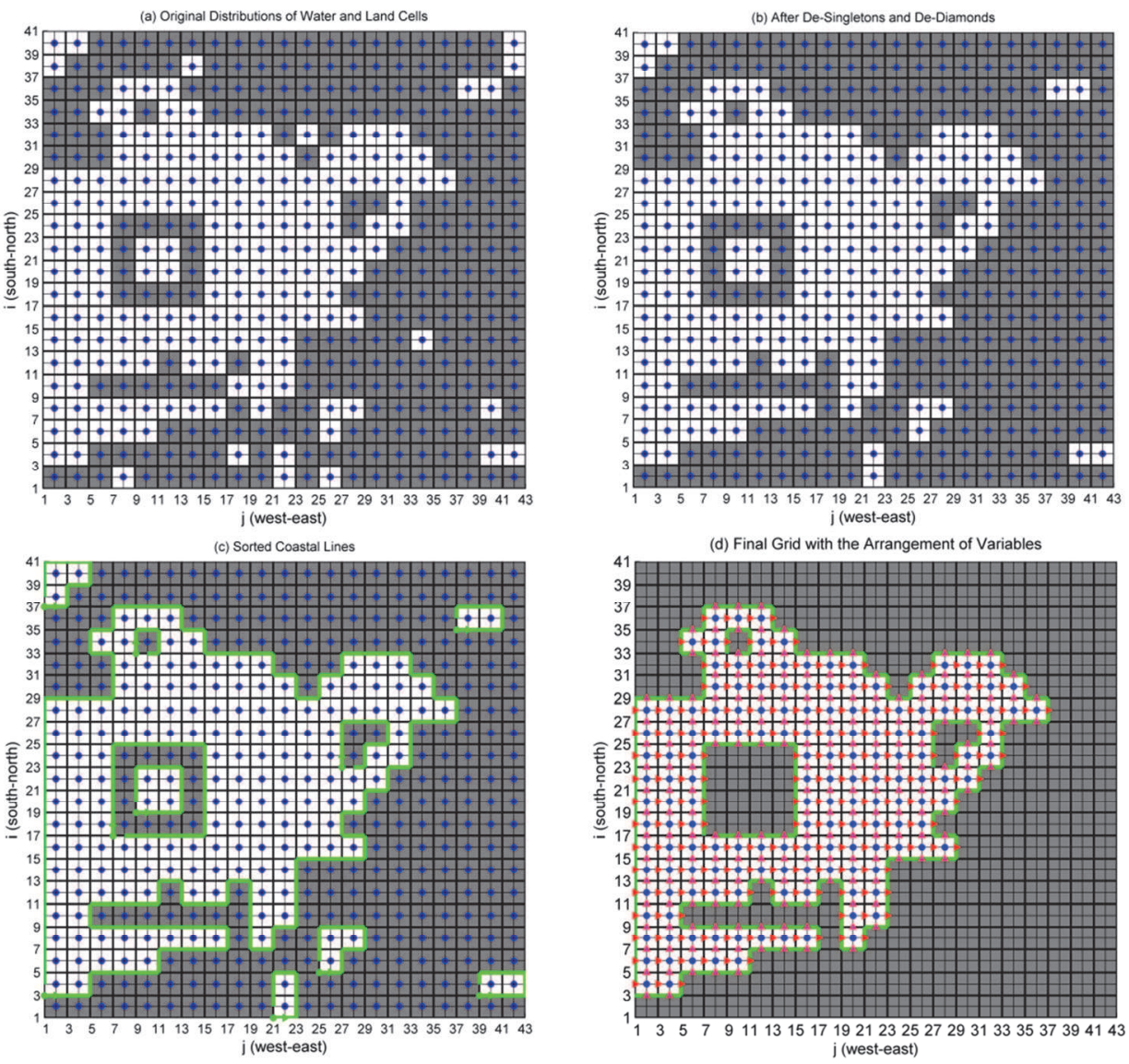

Fig. 1. Procedure from a topographic dataset to a model grid. Panel (a) shows the original distributions of water cells and land cells (in gray) in a topographic dataset. Panel (b) shows the results after removal of the singletons and the diamonds. Panel (c) shows the results after sorting out the coastal lines. Panel (d) defines the model domain with model variables arranged on the grid points.

2. Diamonds. A diamond is a pair of water cells connected by a common corner point. The water cells centered at $(i=8, j=16)$ and $(i=10, j=18)$ in Panel (a) of the figure forms a diamond, sharing a corner point at $(i=9, j=17)$. Diamonds are unwanted because they can cause ambiguity in tracing coastal lines. For example, starting from the point of $(i=3, j=1)$, one can easily follow the coastal line until he reaches the point of $(i=9, j=17)$, where he will have an ambiguity in deciding which direction for his next move. It is necessary to trace coastal lines so that we can well define a model domain.

3. Coastal lines. A coastal line consists of a series of common edges shared by water cells and land cells. In order to well define a model domain, we need an algorithm to automatically find out all of the coastal points and sort them into loops. The longest coastline and any others nested in it (excluding those nested doubly or more) form a multiply connected 
region, which defines the model domain. Water cells that are outside of the multiply connected region can be disregarded (by turning them into land cells).

4. Lagoons. A lagoon consists of a group of water cells which are connected with themselves only, but not with the rest. The water cells centered at $(i=20, j=10),(i=22$, $\mathrm{j}=10),(\mathrm{i}=21, \mathrm{j}=12)$ and $(\mathrm{i}=22, \mathrm{j}=12)$ is one of the lagoons presented in Panel (a). Retaining lagoons are as undesirable as retaining singletons.

These issues can be well addressed and the auto-generation of organized grid can be achieved. Limited by the volume of the chapter, details of the implementations are omitted. Only the basic procedure is outlined in Figure 1: Panel (a) is what the original topographic data look like. Panel (b) is after removal of singletons and diamonds. Panel (c) is after sorting out all the coastal points. The coastal lines are shown in green. The longest one and those nested in only once form a multiply connected region as the model domain. The coastal line whose starting point is at $(i=19, j=9)$ is excluded. It is nested within a nested coastal line, it is nested doubly. Panel (d) is the final stage where all the grid points that are outside of the multiply connected region are removed, and the model variables are arranged on the grid points, as indicated by the blue dots for $\eta$ and red arrows for $U$ and V. Here the variables are arranged according to Arakawa C-grid arrangement (Arakawa and Lamb, 1977).

All the $\eta^{\prime}$ 's, U's and V's defined on the grid can be viewed as elements of three long vectors, $[\eta],[\mathrm{U}]$ and $[\mathrm{V}]$. The square bracket is used to explicitly indicate that the single letter inside is a vector, but may be dropped off in a context where it is clear that the single letter indicates a vector. From the three vectors, we can also form a super long vector $[\eta \mathrm{U} V]$.

\subsection{An auto-generated grid for the India and Pacific Oceans}

The General Bathymetric Chart of the Oceans $\left(\mathrm{GEBCO}^{2}\right)$ provides a fine global bathymetry dataset of half minute resolution (GEBCO_08). With this dataset and the grid generation method mentioned above, we can automatically generate a model grid for any part of the world oceans. Shown in Figure 2 is a model domain for the India and Pacific Oceans with the grid generated from GEBCO_08 decimated to a 5-minute resolution. For clarity, only the coastal lines are shown. The lines in red are the coastal lines, which define the model domain. The blue lines would be the coastal lines if the lagoons were not removed. Note, according to the lagoon definition given above, parts of other big oceans, such as of the Atlantic Ocean, are also viewed as lagoons, because they are outside of the multiply connected region in question.

There are 3,771,781 $\eta$-points, 3,782,233 U-points, 3,784,476 V-points, and 970 coastal lines. The maximum water depth is $10545 \mathrm{~m}$, and the minimum water depth is set to be $10 \mathrm{~m}$. The mean water depth of the domain is $3806 \mathrm{~m}$. In the figure, the green dots show the DART stations (Deep-ocean Assessment and Reporting of Tsunamis 3 ). The three gray zones are the sponge zones next to the three open water boundaries. Both the DART stations and the gray zones will be used later on. A sponge zone is designed to absorb exiting waves so that the unwanted wave reflections can be reduced to minimum. Such a sponge effect has been customarily achieved by setting up much larger than normal values of frictional parameter values. In this study, however, the sponge effect will be achieved through a twodimensional Lax-Friedriches scheme, more details of which will be given in Section 5.2.

\footnotetext{
2 http://www.gebco.net

${ }^{3}$ http://www.ndbc.noaa.gov/dart/dart.shtml
} 


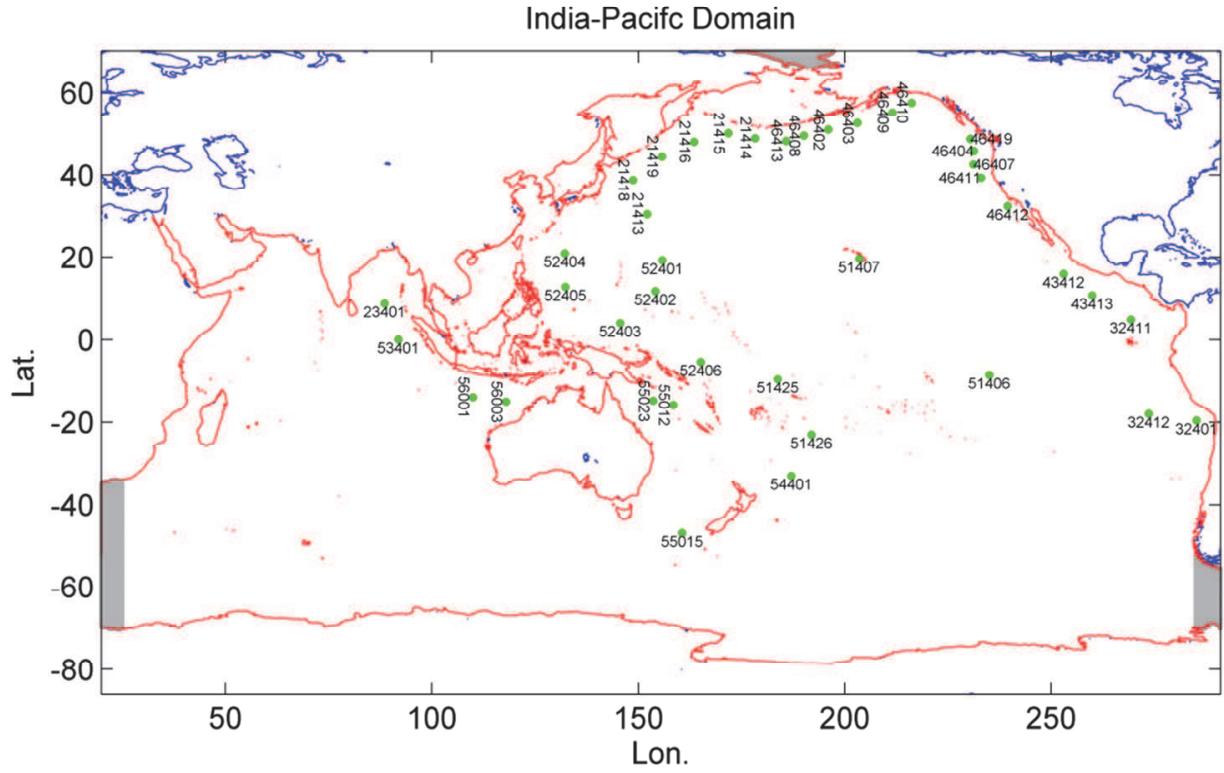

Fig. 2. An India-Pacific grid auto-generated from GEBCO_08 topographic dataset. The coastal lines in red define the model domain. The DART stations are indentified by the green dots and their station numbers. The three gray zones are sponge zones next to the three open water boundaries.

\subsection{K-indexes of model variables}

The model variables defined on the grid points can have their own indexes. For example, there are 157 n-points in Panel (d) of Figure 1, and they can be numbered from 1 to 157, counted from the lower left corner upwards and rightward. Therefore, the first $\eta$ is at $(i=4$, $j=2)$ and the second one is at $(i=6, j=2)$, and the last one is at $(i=28, j=36)$. We may use symbols $k_{e}, k_{u}$ and $k_{v}$ to denote the linear indexes of the variables $\eta, U$ and $V$. respectively. In a context where there is no ambiguity, the subscripts with the k's may be dropped off.

Therefore a variable defined on a grid point can be addressed either through an $(i, j)$-index or a k-index. Obviously there is one-to-one relationship between the two indexing systems. Their conversions will be needed in the next section. Thus let us assume here that the following three index conversion functions have been made:

$$
\begin{aligned}
& \mathrm{ke}_{\mathrm{e}}=\operatorname{KeIndex}(\mathrm{i}, \mathrm{j}) \\
& \mathrm{k}_{\mathrm{u}}=\operatorname{KuIndex}(\mathrm{i}, \mathrm{j}) \\
& \mathrm{k}_{\mathrm{v}}=\operatorname{Kv} \operatorname{Index}(\mathrm{i}, \mathrm{j})
\end{aligned}
$$

which convert the $(i, j)$-indexes to the $k$-indexes. These functions are easy to make, so the details on their implementation are omitted here. 


\section{Global operators}

This section will discuss how to assemble the global operators. The global operators, which come as sparse matrices, will be integrated into the sub-matrices of the dynamic matrix $\mathrm{A}$ as in Eq.(11).

\subsection{Sea surface gradient operators, $G_{x}$ and $G_{y}$}

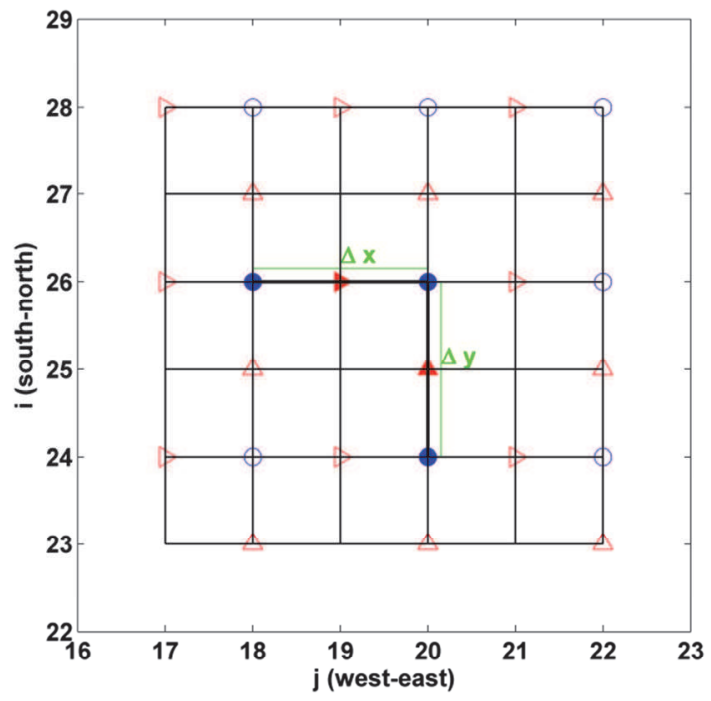

Fig. 3. Grid stencil for consideration of the sea surface gradients. The grid points indicated by the horizontal arrows are the U-points, those indicated by the vertical arrows are the Vpoints, and those by the blue circles are the $\eta$-points.

Extracted from Panel (d) of Figure 1, Figure 3 serves a grid stencil to construct the operators for the sea surface gradients. The $x$-directional sea surface gradient at the point $(i, j)$ for an interior and non coastal U-point, indicated by the solid horizontal red arrow in the figure, can be approximated as

$$
\left.\frac{\mathrm{gh}}{\mathrm{R} \cos \phi} \frac{\partial \eta}{\partial \lambda}\right|_{\mathrm{i}, \mathrm{j}} \approx \frac{g \mathrm{~h}_{\mathrm{i}, \mathrm{j}}}{\Delta \mathrm{x}_{\mathrm{i}, \mathrm{j}}}\left[\begin{array}{ll}
-1 & 1
\end{array}\right]\left[\begin{array}{l}
\eta_{\mathrm{i}, \mathrm{j}-1} \\
\eta_{\mathrm{i}, \mathrm{j}+1}
\end{array}\right]
$$

where $\Delta x_{i, j}=x(i, j+1)-x(i, j-1)$ with $x=R \lambda \cos \phi$. Note that coastal U-points have been excluded from consideration. We need not to assign a force for a coastal U-point, because the value there should be zero all the time.

The coefficient row vector on the RHS of the equation is a local operator, since it operates only on the two neighbouring $\eta$-points. We need to assemble it into a global operator. With aid of the index conversion functions given by Eqs. (12) to (14), we can have

$$
\mathrm{G}_{\mathrm{x}}\left(\mathrm{k}_{0},\left[\mathrm{k}_{1} \mathrm{k}_{2}\right]\right)=\frac{\mathrm{gh}_{\mathrm{i}, \mathrm{j}}}{\Delta \mathrm{x}_{\mathrm{i}, \mathrm{j}}}\left[\begin{array}{ll}
-1 & 1
\end{array}\right]
$$


where $\mathrm{k}_{0}=\operatorname{KuIndex}(\mathrm{i}, \mathrm{j})$ and $\mathrm{k}_{1,2}=\operatorname{KeIndex}(\mathrm{i}, \mathrm{j} \mp 1)$. Thus $\mathrm{G}_{\mathrm{x}}$ is a global gradient operator, which upon operating on the elevation column vector [n], produces a column vector of sea level gradient in the $\mathrm{x}$-direction. Here the word $x$-direction is synonymous to the longitudinal direction.

Matlab users should avoid looping through individual (i, j)-points for the assembly. Vectorizing the $(i, j)$-indexes will greatly enhance the speed of the assembly. Also $G_{x}$ should be treated as a sparse matrix, and use the following syntax in calling the Matlab sparse function for the assembly:

$$
\begin{gathered}
G_{x}=G_{x}+\operatorname{sparse}\left(k_{0}, k_{1}, \frac{-g h_{i, j}}{\Delta x_{i, j}}, \text { kumax, kemax }\right) \\
G_{x}=G_{x}+\operatorname{sparse}\left(k_{0}, k_{2}, \frac{g h_{i, j}}{\Delta x_{i, j}}, \text { kumax, kemax }\right)
\end{gathered}
$$

where kumax and kemax means the maximums of $\mathrm{k}_{\mathrm{u}^{-}}$and $\mathrm{k}_{\mathrm{v}}$-indexes. This syntax avoids indexing into $G_{x}$ on the LHS of the equation for the sake of a big difference in the computational speed. ${ }^{4}$ If $\mathrm{G}_{\mathrm{x}}$ starts to be a zero sparse matrix, all the entries will be accumulated correctly. The same advices apply to the assembly of the other global operators to be discussed below.

The $y$-directional sea surface gradient at the point $(i, j)$ for an interior and non-coastal Vpoint, indicated this time by the solid vertical red arrow in Figure 3, can be approximated by

$$
\left.\frac{\operatorname{gh}}{\mathrm{R}} \frac{\partial \eta}{\partial \phi}\right|_{\mathrm{i}, \mathrm{j}} \approx \frac{\mathrm{gh}_{\mathrm{i}, \mathrm{j}}}{\Delta \mathrm{y}_{\mathrm{i}, \mathrm{j}}}\left[\begin{array}{ll}
-1 & 1
\end{array}\right]\left[\begin{array}{l}
\eta_{\mathrm{i}-1, \mathrm{j}} \\
\eta_{\mathrm{i}+1, \mathrm{j}}
\end{array}\right]
$$

where $\mathrm{y}=\mathrm{R} \phi$, and the local operator the RHS can be the assembled in to a global one as

$$
\mathrm{G}_{\mathrm{y}}\left(\mathrm{k}_{0},\left[\mathrm{k}_{1} \mathrm{k}_{2}\right]\right)=\frac{\mathrm{gh}_{\mathrm{i}, \mathrm{j}}}{\Delta \mathrm{y}_{\mathrm{i}, \mathrm{j}}}\left[\begin{array}{ll}
-1 & 1
\end{array}\right]
$$

where $\mathrm{k}_{0}=\operatorname{KvIndex}(\mathrm{i}, \mathrm{j})$ and $\mathrm{k}_{1,2}=\operatorname{KeIndex}(\mathrm{i} \mp 1, \mathrm{j}) \cdot \mathrm{G}_{\mathrm{y}}$ is global operator, when it operates

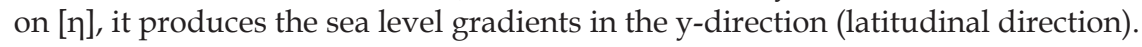

\subsection{Mass divergence operators, $D_{x}$ and $D_{y}$}

Figure 4 gives a cell for consideration of the mass divergence through the cell. An approximation for the mass divergence at $(i, j)$ due to the fluxes associated with $U$ is

$$
\left.\frac{\partial \mathrm{U}}{\partial \mathrm{x}}\right|_{\mathrm{i}, \mathrm{j}} \approx\left[-\frac{\Delta \mathrm{y}_{\mathrm{i}, \mathrm{j}}}{\mathrm{e}} \frac{\Delta \mathrm{y}_{\mathrm{i}, \mathrm{j}}}{\mathrm{e}}\right]\left[\begin{array}{l}
\mathrm{U}_{\mathrm{i}, \mathrm{j}-1} \\
\mathrm{U}_{\mathrm{i}, \mathrm{j}+1}
\end{array}\right]
$$

where $\mathrm{x}=\mathrm{R} \lambda \cos \phi$, and $\Delta \mathrm{y}_{\mathrm{i}, \mathrm{j}}=\mathrm{y}_{\mathrm{i}+1, \mathrm{j}}-\mathrm{y}_{\mathrm{i}-1, \mathrm{j}}$ with $\mathrm{y}=\mathrm{R} \phi$, and

$$
\mathrm{e}=2 \mathrm{R}^{2}\left(\lambda_{\mathrm{e}}-\lambda_{\mathrm{w}}\right) \cos \frac{\phi_{\mathrm{n}}+\phi_{\mathrm{s}}}{2} \sin \frac{\phi_{\mathrm{n}}-\phi_{\mathrm{s}}}{2}
$$

\footnotetext{
${ }^{4}$ http:/ / blogs.mathworks.com/loren/2007/03/01/creating-sparse-finite-element-matrices-in-matlab/
} 


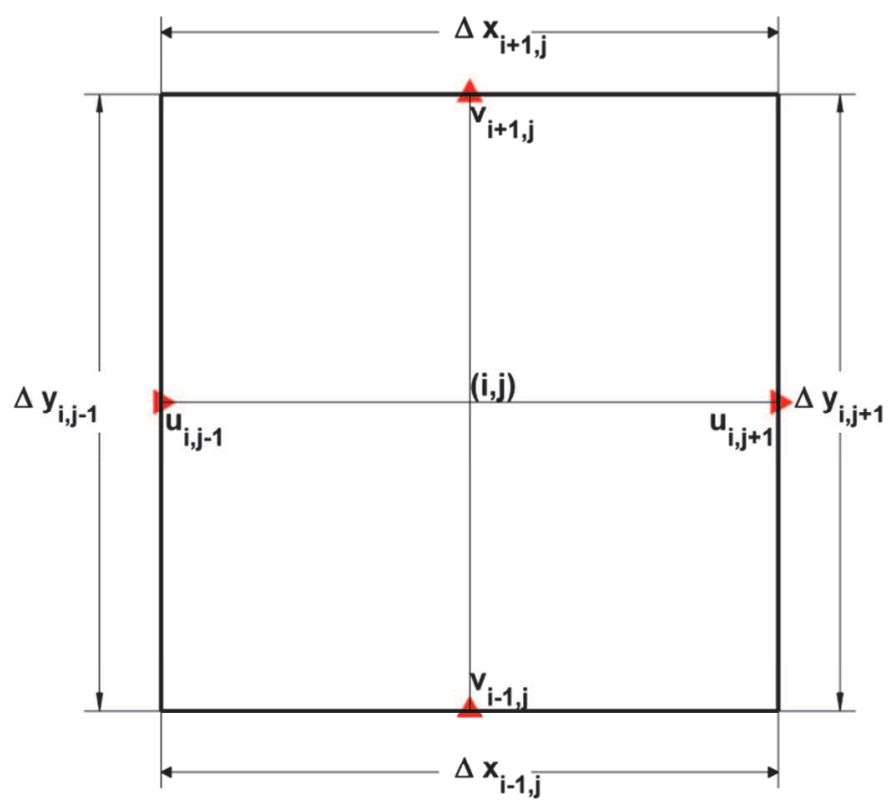

Fig. 4. A cell for the consideration of water mass divergence. The west and east side lengths of the cell are denoted by $\Delta y_{\mathrm{i}, \mathrm{j}-1}$ and $\Delta y_{\mathrm{i}, \mathrm{j}+1}$, which are equal. The south and north side lengths are denoted by $\Delta x_{i-1, j}$ and $\Delta x_{i+1, j}$ which are not equal on a spherical surface. The streams normal to the four sides are denoted by $U_{i, j-1}, U_{i, j+1}, V_{i-1, j}$, and $V_{i+1, j}$.

is the area of the cell. The 2-element row vector on the RHS of Eq.(21) is a local mass divergence operator, which operates on the two neighbouring U's and produces a cell-mean mass divergence due to the fluxes through the west and east sides of the cell. The mass divergence at $(i, j)$ due to fluxes caused by $\mathrm{V}$ can be approximated by

$$
\left.\frac{1}{R \cos \phi} \frac{\partial \mathrm{V} \cos \phi}{\partial \phi}\right|_{\mathrm{i}, \mathrm{j}} \approx\left[-\frac{\Delta \mathrm{x}_{\mathrm{i}-1, \mathrm{j}}}{\mathrm{e}} \frac{\Delta \mathrm{x}_{\mathrm{i}+1, \mathrm{j}}}{\mathrm{e}}\right]\left[\begin{array}{c}
\mathrm{V}_{\mathrm{i}-1, \mathrm{j}} \\
\mathrm{V}_{\mathrm{i}+1, \mathrm{j}}
\end{array}\right]
$$

where $\Delta x_{i \pm 1, j}=x(i \pm 1, j+1)-x(i \pm 1, j-1)$ are the lengths of the north and south sides of the cell. The 2-element row vector on the RHS of Eq.(23) is another local mass divergence operator, which operates on the two neighbouring V's and produces a cell-mean mass divergence due to the fluxes through the south and north sides of the cell.

The two local divergence operators need to be assembled into their global counterparts

$$
\begin{aligned}
& \mathrm{D}_{\mathrm{x}}\left(\mathrm{k}_{0},\left[\mathrm{k}_{1} \mathrm{k}_{2}\right]\right)=\left[-\frac{\Delta \mathrm{y}_{\mathrm{i}, \mathrm{j}}}{\mathrm{e}} \frac{\Delta \mathrm{y}_{\mathrm{i}, \mathrm{j}}}{\mathrm{e}}\right] \\
& \mathrm{D}_{\mathrm{y}}\left(\mathrm{k}_{0},\left[\mathrm{k}_{3} \mathrm{k}_{4}\right]\right)=\left[-\frac{\Delta \mathrm{x}_{\mathrm{i}-1, \mathrm{j}}}{\mathrm{e}} \frac{\Delta \mathrm{x}_{\mathrm{i}+1, \mathrm{j}}}{\mathrm{e}}\right]
\end{aligned}
$$

where $\mathrm{k}_{0}=\operatorname{KeIndex}(\mathrm{i}, \mathrm{j}), \mathrm{k}_{1,2}=\operatorname{KuIndex}(\mathrm{i}, \mathrm{j} \mp 1), \mathrm{k}_{3,4}=\operatorname{KvIndex}(\mathrm{i} \mp 1, \mathrm{j})$ 


\subsection{Coriolis Operator, $\mathrm{C}_{\mathrm{u}}$ and $\mathrm{C}_{\mathrm{v}}$}

Since the staggered Arakawa C-grid does not co-locate the U- and V-points, for evaluations of the Coriolis force we have to resort to a kind of mean values of U's and V's. For an interior Vpoint, which is neither on open water boundary nor on coast, there are always four next neighbouring U-points. The four neighbouring U's can be somehow averaged and the result, denoted as $\overline{\mathrm{U}}$, can then be used for the evaluation of the Coriolis force, $f \overline{\mathrm{U}}$, at the V-point.

As often seen, a straight forward way to calculate an $\bar{U}$ is to sum over the four surrounding U's and then divide the result by 4. However, Espelid et al (2000) pointed out that such an equally weighted averaging could result in an artificial energy growth or decay in an environment where the Coriolis parameter and/or the water depth varied spatially. To see this point, we can first multiply Eqs.(2) and (3) by $\mathrm{U} / \mathrm{h}$ and $\mathrm{V} / \mathrm{h}$ respectively and then add them to have an equation for the kinetic energy over the water column. In doing so, we can see that the contributions to the kinetic energy by the Coriolis force in the two directions cancel each other: $\frac{f V U}{h}-\frac{f U V}{h}=0$, a well known property in geo-fluid dynamics that Coriolis force does not do any work. However the spatial discretization can destroy this property. On a staggered grid such as the C-grid where U and V-points are not collocated, the two terms may not cancel each other because they are not evaluated at the same point, giving rise to increasing or decaying in energy artificially.

As a remedy to this problem, Espelid et al (2000) proposed the following weighted average scheme,

$$
\left.\overline{\mathrm{U}}\right|_{i, j}=\frac{w_{i, j}}{4} \sum_{m=j-1}^{m=j+1} \sum_{l=i-1}^{l=i+1} \frac{U_{l, m}}{w_{l, m}}
$$

where $w_{i, j}=\sqrt{h_{i, j} /\left|f_{i . j}\right|}$ is the weight for the averaging. The weighted average $\bar{V}$ can be calculated in the same way. With this averaging scheme, one can verify that the work done by the two Coriolis force components cancel each other point wise in the C-grid, if the Coriolis parameter does not change its sign. It is interesting to note that the $1 / w_{i, j}$ is the square-root of the planetary vorticity, f/h, (e.g. Pedloski, 1979), which is also known as the geostrophic contours. In the absence of the external force and internal friction, the circulations in the ocean tend to flow the geostrophic contours.

However it seems that the above scheme was designed only for a regional model where there is no need to be concerned with sign changes of the Coriolis parameter. For a global mode, or a regional model whose domain is across the equator, or a model whose spherical north pole is shifted on a land to avoid a mathematical singularity which otherwise would arise at the geographic north pole, we do need to be concerned with the case where the discretized Coriolis parameters, $f_{i, j}$, at the four neighbouring points do not shear the same signs. To address this concern, the averaging weight should be modified as $w_{i, j}=\operatorname{sgn}\left(f_{i, j}\right) \sqrt{h_{i, j} /\left|f_{i . j}\right|}$. Therefore, with the modified averaging weight, we can have the following formula to evaluate the Coriolis force at a V-point,

$$
(f \bar{U})_{i, j}=\frac{\sqrt{\left|\mathrm{f}_{\mathrm{i}, \mathrm{j}}\right| \mathrm{h}_{\mathrm{i}, \mathrm{j}}}}{4}\left[\frac{\frac{\mathrm{s}_{1} \sqrt{\left|\mathrm{f}_{\mathrm{i}-1, \mathrm{j}-1}\right|}}{\sqrt{\mathrm{h}_{\mathrm{i}-1, \mathrm{j}-1}}}}{\frac{\mathrm{s}_{2} \sqrt{\left|\mathrm{f}_{\mathrm{i}+1, \mathrm{j}-1}\right|}}{\sqrt{\mathrm{h}_{\mathrm{i}+1, \mathrm{j}-1}}}} \frac{\mathrm{s}_{3} \sqrt{\left|\mathrm{f}_{\mathrm{i}-1, \mathrm{j}+1}\right|}}{\sqrt{\mathrm{h}_{\mathrm{i}-1, \mathrm{j}+1}}} \frac{\mathrm{s}_{4} \sqrt{\left|\mathrm{f}_{\mathrm{i}+1, \mathrm{j}+1}\right|}}{\sqrt{\mathrm{h}_{\mathrm{i}+1, \mathrm{j}+1}}}\right]\left[\begin{array}{l}
\mathrm{U}_{\mathrm{i}-1, \mathrm{j}-1} \\
\mathrm{U}_{\mathrm{i}+1, \mathrm{j}-1} \\
\mathrm{U}_{\mathrm{i}-1, \mathrm{j}+1} \\
\mathrm{U}_{\mathrm{i}+1, \mathrm{j}-1}
\end{array}\right]
$$


where $s_{1}=\operatorname{sgn}\left(f_{i-1, j-1}\right), s_{2}=\operatorname{sgn}\left(f_{i+1, j-1}\right), s_{3}=\operatorname{sgn}\left(f_{i-1, j+1}\right)$ and $s_{4}=\operatorname{sgn}\left(f_{i+1, j+1}\right)$. The 4element row vector on the RHS is a local Coriolis operator, which operates on the neighbouring U's and produces Coriolis force at the center V-point. We need to assemble this local Coriolis operator into its global counterpart $\mathrm{C}_{\mathrm{u}}$, as

$$
\begin{aligned}
& \mathrm{C}_{\mathrm{u}}\left(\mathrm{k}_{0},\left[\mathrm{k}_{1} \mathrm{k}_{2} \mathrm{k}_{3} \mathrm{k}_{4}\right]\right) \\
& =\frac{\sqrt{\left|\mathrm{f}_{\mathrm{i}, \mathrm{j}}\right| \mathrm{h}_{\mathrm{i}, \mathrm{j}}}}{4}\left[\frac{\mathrm{s}_{1} \sqrt{\left|\mathrm{f}_{\mathrm{i}-1, \mathrm{j}-1}\right|}}{\sqrt{\mathrm{h}_{\mathrm{i}-1, \mathrm{j}-1}}} \frac{\mathrm{s}_{2} \sqrt{\left|\mathrm{f}_{\mathrm{i}+1, \mathrm{j}-1}\right|}}{\sqrt{\mathrm{h}_{\mathrm{i}+1, \mathrm{j}-1}}} \frac{\mathrm{s}_{3} \sqrt{\left|\mathrm{f}_{\mathrm{i}-1, \mathrm{j}+1}\right|}}{\sqrt{\mathrm{h}_{\mathrm{i}-1, \mathrm{j}+1}}} \frac{\mathrm{s}_{4} \sqrt{\left|\mathrm{f}_{\mathrm{i}+1, \mathrm{j}+1}\right|}}{\sqrt{\mathrm{h}_{\mathrm{i}+1, \mathrm{j}+1}}}\right]
\end{aligned}
$$

with $\mathrm{k}_{0}=\operatorname{KvIndex}(\mathrm{i}, \mathrm{j}), \mathrm{k}_{1,2}=\operatorname{KuIndex}(\mathrm{i} \mp 1, \mathrm{j}-1), \mathrm{k}_{3,4}=\operatorname{KuIndex}(\mathrm{i} \mp 1, \mathrm{j}+1)$.

When the positions of the two indexing functions, KvIndex and KuIndex, in the above are switched, Eq.(28) can be equally well used to calculate $\mathrm{C}_{\mathrm{v}}$, another global Coriolis operator which operates on the $\mathrm{V}$-variables and produced the Coriolis force on all the U-points.

\subsection{Frictional operators, $F_{u}$ and $F_{v}$}

The frictional operators are easiest to assemble. They are diagonal, with elements being $\mathrm{k} / \mathrm{h}$. Let $\mathrm{F}_{u}$ denote a global frictional operator, which operates on $\mathrm{U}$ and produces frictional force in $\mathrm{x}$-direction. It can be given by

$$
\mathrm{F}_{\mathrm{u}}(\mathrm{k}, \mathrm{k})=\kappa_{\mathrm{i}, \mathrm{j}} / \mathrm{h}_{\mathrm{i}, \mathrm{j}}
$$

with $\mathrm{k}=\operatorname{KuIndex}(\mathrm{i}, \mathrm{j})$ for a $(\mathrm{i}, \mathrm{j})$-point where $\mathrm{U}$ is defined. Similarly we can have a frictional operator $\mathrm{F}_{u}$ which operates on $\mathrm{V}$ and produces frictional force in the $\mathrm{y}$-direction,

$$
\mathrm{F}_{\mathrm{v}}(\mathrm{k}, \mathrm{k})=\kappa_{\mathrm{i}, \mathrm{j}} / \mathrm{h}_{\mathrm{i}, \mathrm{j}}
$$

with $k=\operatorname{KvIndex}(i, j)$ for $a(i, j)$-point where $V$ is defined.

\subsection{Open boundary operators $\mathbb{O}_{\mathrm{u}}$ and $\mathbb{O}_{\mathrm{v}}$}

Since the most outside variable points are velocity points, the Sommerfeld radiation boundary condition are thus applied at the U- and V-points on open water boundaries. Substitute $\mathrm{U}$ and $\mathrm{V}$ for $\mathrm{p}$ in Eq.(7), we can have

where $c=\sqrt{\text { gh }}$.

$$
\begin{array}{lc}
\frac{\partial \mathrm{U}}{\partial \mathrm{t}}+\mathrm{c} \frac{\partial \mathrm{U}}{\partial \mathrm{n}}=0 & \text { at east and west open water boundaries } \\
\frac{\partial \mathrm{V}}{\partial \mathrm{t}}+\mathrm{c} \frac{\partial \mathrm{V}}{\partial \mathrm{n}}=0 & \text { at south and north water boundaries }
\end{array}
$$

For a $\mathrm{U}_{\mathrm{i}, \mathrm{j}}$ on an open water boundary, its outward and normal derivative can be approximated as,

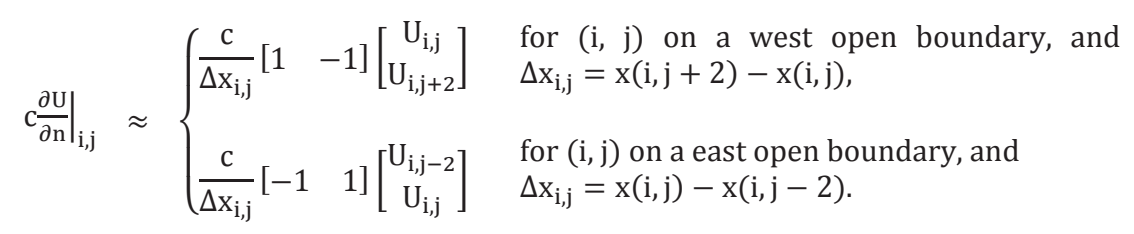

where $c=\sqrt{g h_{i, j}}$. Similarly, for a $V_{i, j}$ on an open water boundary, 


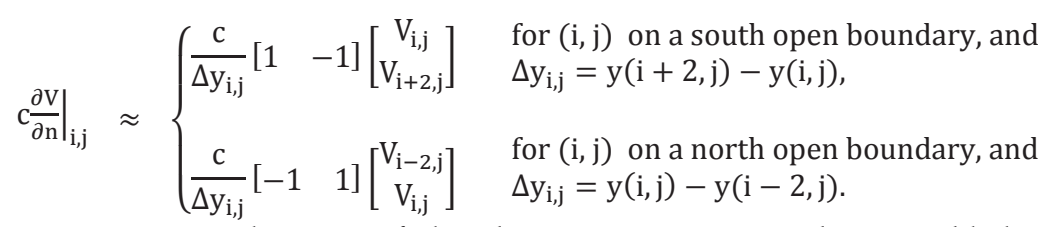

The row vectors on the RHS of the above expressions can be assembled into a global operator $\mathbb{O}_{\mathrm{u}}$ as

$$
\mathbb{O}_{\mathrm{u}}\left(\mathrm{k}_{0}, \mathrm{k}_{1}\right)=\frac{\mathrm{c}}{\Delta \mathrm{x}_{\mathrm{i}, \mathrm{j}}}\left[\begin{array}{ll}
1 & -1
\end{array}\right]
$$

with $\mathrm{k}_{0}=\operatorname{KuIndex}(\mathrm{i}, \mathrm{j})$ and $\mathrm{k}_{1}=\operatorname{KuIndex}(\mathrm{i}, \mathrm{j}+2)$, and

$$
\mathbb{O}_{\mathrm{u}}\left(\mathrm{k}_{0}, \mathrm{k}_{1}\right)=\frac{\mathrm{c}}{\Delta \mathrm{x}_{\mathrm{i}, \mathrm{j}}}\left[\begin{array}{ll}
-1 & 1
\end{array}\right]
$$

with $\mathrm{k}_{0}=\operatorname{KuIndex}(\mathrm{i}, \mathrm{j}-2)$ and $\mathrm{k}_{1}=\operatorname{Ku} \operatorname{Index}(\mathrm{i}, \mathrm{j})$ when point $(\mathrm{i}, \mathrm{j})$ is on a east open boundary. Upon operating on the U-vector, $\mathbb{O}_{\mathrm{u}}$ produces a vector of outward gradients in $\mathrm{U}$ along the east and west open boundaries.

$\mathbb{O}_{\mathrm{v}}$ can be assembled in the save way but with the KvIndex function instead.

\section{Linear shallow water equations in matrix form}

All the operators constructed in the proceeding section can now be put together to discretize in space the governing equations and boundary conditions for the linear shallow water dynamics. We also have to be concerned with how to discretize the equation in time, and how to solve the total discretized equations in a manner that is computationally stable. These issues are the content of this section.

\subsection{Governing equations and boundary conditions in one matrix equation}

With the global operators constructed in the proceeding section, we can now discretize the RHS of Eqs.(1) to (3) and Eqs (5) to (7), and present the result in a single matrix equation as follows:

$$
\frac{\partial}{\partial t}\left[\begin{array}{l}
\eta_{1} \\
\mathrm{U} \\
\mathrm{V}
\end{array}\right]=\left[\begin{array}{ccc}
0 & -\mathrm{D}_{x} & -\mathrm{D}_{y} \\
-\mathrm{G}_{x} & -\mathbb{O}_{u}-\mathrm{F}_{\mathrm{u}} & \mathrm{C}_{\mathrm{v}} \\
-\mathrm{G}_{y} & -\mathrm{C}_{\mathrm{u}} & -\mathbb{O}_{v}-\mathrm{F}_{\mathrm{v}}
\end{array}\right]\left[\begin{array}{c}
\eta \\
\mathrm{U} \\
\mathrm{V}
\end{array}\right]
$$

where 0 represents a zero matrix.

The zero normal flow conditions at coasts have accommodated in the U- and V- vectors. All the stream points on the coasts are included as the elements of the two stream vectors. The coastal elements start to be zero and will remain to be so. They are guaranteed to remain to be zero because $\mathrm{F}_{\mathrm{u}}$ and $\mathrm{F}_{\mathrm{v}}$ are diagonal, whereas the corresponding rows of the other submatrices are all zero. In fact, the coastal elements can be deleted from the U- and V-vectors, and the related sub-matrices can shrink just to include the interior and open boundary points in order to reduce the computations. This can be automatically cared within a program running the matrix-vector multiplications. Conceptually however, we had better regard that the U- and V-vectors include their coastal elements. The radiation conditions at open water boundaries are implemented through $\mathbb{O}_{\mathrm{u}}$ and $\mathbb{O}_{\mathrm{v}}$. In case there are no open water boundaries, they become zero matrices. 


\subsection{Time discretization schemes and sponge zones}

The time derivatives remain to be discretized, for which we have two schemes. Take the discretization for $\partial \mathrm{p} / \partial \mathrm{t}$ as example, where $\mathrm{p}$ may represent $\eta, \mathrm{U}$, or $\mathrm{V}$, we can have

$$
\left.\frac{\partial \mathrm{p}}{\partial \mathrm{t}}\right|_{\mathrm{i}, \mathrm{j}} \approx \frac{\mathrm{p}_{\mathrm{i}, \mathrm{j}}^{(\tau+1)}-\mathrm{p}_{\mathrm{i}, \mathrm{j}}^{(\tau)}}{\Delta \mathrm{t}}, \quad(\mathrm{p}=\eta, \mathrm{U} \text { or } \mathrm{V})
$$

or

$$
\left.\frac{\partial \mathrm{p}}{\partial \mathrm{t}}\right|_{\mathrm{i}, \mathrm{j}} \approx \frac{\mathrm{p}_{\mathrm{i}, \mathrm{j}}^{(\tau+1)}-\overline{\mathrm{p}}_{\mathrm{i}, \mathrm{j}}^{(\tau)}}{\Delta \mathrm{t}}, \quad(\mathrm{p}=\eta, \mathrm{U} \text { or } \mathrm{V})
$$

where $\Delta t$ is the time step, the superscript $\tau$ is a time step index such that $t=\tau \Delta$ t for $\tau=$ $0,1,2, \cdots$. The second scheme differs from the first one in its second term, where an averaged elevation, $\bar{p}_{i, j}$ instead of $p_{i, j}$, is used. For variable on an interior grid point $(i, j)$, the average is defined as follows:

$$
\bar{p}_{i, j}=\left\{\begin{array}{cl}
\frac{p_{i, j-2}+p_{i, j+2}+p_{i-2, j}+p_{i+2, j}}{4} & \begin{array}{c}
\text { if } 2 \text { pairs of neighbours which are } \\
\text { non-boundary nodes. }
\end{array} \\
\mathrm{p}_{\mathrm{i}, \mathrm{j}} & \text { otherwise, no averaging. }
\end{array}\right.
$$

In other words, $\overline{\mathrm{p}}_{\mathrm{i}, \mathrm{j}}$ is obtained by averaging over possible neighbours. The neighbours here are all in the sense of two grid-point apart, i.e., in the sense of $i \pm 2$, and/or $j \pm 2$. The neighbours must also be of the same types, e.g., the neighbours of an $\eta$-point must be $\eta$ points too. The neighbours have to be aligned either in the i-direction or in the j-direction. Most interior points have two lined pairs, such as those away from coasts. For an interior point near a coast, it may have one or zero lined pairs. In Figure 1, the $\eta$-point at $(i=28, j=34)$ has three neighbouring $\eta$-points: $(i=28, j=32),(i=28, j=36)$, and $(i=30, j=34)$, and only the first two points forms a lined pair. The $\eta$-point at $(i=24, j=32)$ has zero lined pair, because its two neighbours at $(i=24, j=30)$ and $(i=26, j=32)$ are not aligned.

The above averaging scheme has excluded certain types of points: Firstly only the interior points are assigned with the averages, the boundary nodes do not need the averages. This exclusion assures that the condition of zero normal flow at coasts will not be destroyed. Secondly, in assigning an average for an interior point, its boundary neighbours are always excluded even when they are part of lined neighbours. This exclusion is to avoid a zero averaged value at a place where the actual value should not. The averaged $U$ at $(i=24, j=31)$ would be zero if its four coastal neighbours were not excluded. Thirdly, open water boundary nodes are also excluded from averaging. Updating on the open boundary nodes is cared by the radiation boundary conditions.

Scheme (39) is an extension of the well known Lax-Friedrichs scheme in one dimension to the two dimensional case here. It helps to stabilize the computation, because it equivalently introduces a horizontal frictional term. To see this point, let us first note the following identities and approximations

$$
\begin{aligned}
\frac{\mathrm{p}_{\mathrm{i}, \mathrm{j}}^{(\tau+1)}-\overline{\mathrm{p}}_{\mathrm{i}, \mathrm{j}}^{(\tau)}}{\Delta \mathrm{t}} & \equiv \frac{\mathrm{p}_{\mathrm{i}, \mathrm{j}}^{(\tau+1)}-\mathrm{P}_{\mathrm{i}, \mathrm{j}}^{(\tau)}-\left(\overline{\mathrm{p}}_{\mathrm{i}, \mathrm{j}}^{(\tau)}-\mathrm{P}_{\mathrm{i}, \mathrm{j}}^{(\tau)}\right)}{\Delta \mathrm{t}} \\
& \equiv \frac{\mathrm{p}_{\mathrm{i}, \mathrm{j}}^{(\tau+1)}-\mathrm{P}_{\mathrm{i}, \mathrm{j}}^{(\tau)}}{\Delta t}-\left(\frac{\Delta x^{2}}{4 \Delta t}\right) \frac{\mathrm{p}_{\mathrm{i}, \mathrm{j}+1}^{(\tau)}-2 \mathrm{p}_{\mathrm{i}, \mathrm{j}}^{(\tau)}+\mathrm{p}_{\mathrm{i}, \mathrm{j}-1}^{(\tau)}}{\Delta x^{2}}-\left(\frac{\Delta y^{2}}{4 \Delta t}\right) \frac{\mathrm{p}_{\mathrm{i}+1, \mathrm{j}}^{(\tau)}-2 \mathrm{p}_{\mathrm{i}, \mathrm{j}}^{(\tau)}+\mathrm{p}_{\mathrm{i}-1, \mathrm{j}}^{(\tau)}}{\Delta y^{2}}
\end{aligned}
$$




$$
\approx \frac{\partial \mathrm{p}}{\partial \mathrm{t}}-\left(\frac{\Delta \mathrm{x}^{2}}{4 \Delta \mathrm{t}}\right) \frac{\partial^{2} \mathrm{p}}{\partial \mathrm{x}^{2}}-\left(\frac{\Delta \mathrm{y}^{2}}{4 \Delta \mathrm{t}}\right) \frac{\partial^{2} \mathrm{p}}{\partial \mathrm{y}^{2}}
$$

Now the time difference has been the split into three terms. The first term approximates the time derivative, the second and the third terms resemble the horizontal momentum diffusion terms, with the $\left(\frac{\Delta x^{2}}{4 \Delta t}\right)$ and $\left(\frac{\Delta y^{2}}{4 \Delta t}\right)$ being the diffusivities in the $x$ - and $y$-directions when $\mathrm{p}$ stands for $\mathrm{U}$ and $\mathrm{V}$ respectively. We started with a set of governing equations, i.e., Eqs (1), (2) and (3), which left out the horizontal friction, now consideration for the computational stabilization has led us to re-pick up what was left out.

However numerical experiments with the India-Pacific grid show that two dimensional LaxFriedrich scheme brings in too strong numerical dissipations. It not only desirably suppresses the small scales noises, but also undesirably smears out or even kills wave forms. Thus scheme (39) will only be used as a complement to scheme (38). We need two parameters to reflect the proportionality of the two schemes. Let $\alpha, \beta \in[0,1]$, and $\alpha+\beta=1$, the two schemes can be joined together as

$$
\left.\frac{\partial \mathrm{p}}{\partial \mathrm{t}}\right|_{\mathrm{i}, \mathrm{j}}=\frac{\mathrm{p}_{\mathrm{i}, \mathrm{j}}^{(\tau+1)}-\alpha \mathrm{p}_{\mathrm{i}, \mathrm{j}}^{(\tau)}-\beta \overline{\mathrm{p}}_{\mathrm{i}, \mathrm{j}}^{(\tau)}}{\Delta \mathrm{t}}
$$

When $\alpha$ is $1, \beta$ is 0 and the joined scheme recovers scheme (38); when $\alpha$ is $0, \beta$ is 1 and the joined scheme recovers scheme (39). Note that $\alpha$ and $\beta$ are not independent. They are both introduced just for brevity of the mathematical expressions that involve them.

The averaging scheme as in Eq.(40) has to be assembled into three global averaging operators, $Q_{e}, Q_{u}$ and $Q_{v}$ for $p=\eta, U$ or $V$. This can be done in the same way as for assembling the other global operators. Replacing $\mathrm{p}$ respectively by vectors $\eta, U$ and $V$, we can now express Eq. (44) in matrix form,

$$
\begin{gathered}
\frac{\partial}{\partial \mathrm{t}} \eta \approx \frac{\eta^{(\tau+1)}-\mathbb{I}_{\mathrm{e}} \eta^{(\tau)}}{\Delta \mathrm{t}} \\
\frac{\partial}{\partial \mathrm{t}} \mathrm{U} \approx \frac{\mathrm{U}^{(\tau+1)}-\mathbb{I}_{\mathrm{u}} \mathrm{U}^{(\tau)}}{\Delta \mathrm{t}} \\
\frac{\partial}{\partial \mathrm{t}} \mathrm{V} \approx \frac{\mathrm{V}^{(\tau+1)}-\mathbb{I}_{\mathrm{V}} \mathrm{V}^{(\tau)}}{\Delta \mathrm{t}}
\end{gathered}
$$

where I's are

$$
\begin{aligned}
& \mathbb{I}_{\mathrm{e}}=\left(\alpha_{\mathrm{e}} \mathrm{I}_{\mathrm{e}}+\beta_{\mathrm{e}} \mathrm{Q}_{\mathrm{e}}\right) \\
& \mathbb{I}_{\mathrm{u}}=\left(\alpha_{\mathrm{u}} \mathrm{I}_{\mathrm{u}}+\beta_{\mathrm{u}} \mathrm{Q}_{\mathrm{u}}\right) \\
& \mathbb{I}_{\mathrm{v}}=\left(\alpha_{\mathrm{v}} \mathrm{I}_{\mathrm{v}}+\beta_{\mathrm{v}} \mathrm{Q}_{\mathrm{v}}\right)
\end{aligned}
$$

and the I's are identity matrices, with their subscripts indicating with which vectors, $\eta$, $U$ or $\mathrm{V}$, their sizes are conformable. The $\alpha$ and $\beta$ parameters are now also presented with appropriate subscripts too. As we can see, the I's are not identity matrices, but are linked to the latter. When $\alpha^{\prime}$ s are 1 and $\beta^{\prime}$ s are 0 , I's become I's. 
Adjusting the value of $\beta$, and hence of $a$, between 0 and 1 , gives a control to balance between the sub-grid noise suppressing and wave form preserving. If let $a$ and $\beta$ be diagonal matrices, we even can have a control point wisely. The balance experiments have been performed in in the India and Pacific domain, and the following are the experiences:

1. Model solutions are very sensitive to the values of $\beta^{\prime}$ s, especially of $\beta_{\mathrm{e}}$. When $\beta^{\prime}$ s are set to be $\geq 0.1$, strong numerical dispassion results; waves die quickly.

2. In the interior region away from the open water boundaries, assigning 0 to $\beta_{\mathrm{e}}$ and 0.005 to both $\beta_{\mathrm{u}}$ and $\beta_{\mathrm{v}}$ can satisfactorily filter out noises at sub-grid scales, but keep information on larger scales almost un-touched.

3. A radiation boundary condition will more or less reflect an outgoing signal back into the model domain (Chapman, 1987), which is undesirable. To suppress this kind of reflections, a sponge zone can set up where large values of $\beta_{\mathrm{e}}, \beta_{\mathrm{u}}, \beta_{\mathrm{v}}$, can be assigned. Values of 0.1 for all $\beta_{\mathrm{e}}, \beta_{\mathrm{u}}$, and $\beta_{\mathrm{v}}$ are large enough. Traditionally a sponge zone means a zone where the frictional parameters (such as $\mathrm{K}$ in the dynamic system considered here) are set up with values much larger than the realistic values. Here the LaxFreidrich's scheme is used as an effective frictional source. The sponge zones shown in Figure 2 are made for this purpose.

Setting $\beta_{u}$ and $\beta_{v}$ to be 0.005 for the interior region is equivalent to setting the horizontal momentum diffusivities to be $0.005\left(\frac{\Delta \mathrm{x}^{2}}{4 \Delta \mathrm{t}}\right)$ and $0.005\left(\frac{\Delta y^{2}}{4 \Delta \mathrm{t}}\right)$. For the India-Pacific grid used for this chapter, which has a 5-min spatial resolution longitude and latitude, $\Delta y=9266 \mathrm{~m}$ and $\Delta x$ varies from $1815 \mathrm{~m}$ to $9266 \mathrm{~m}$. The $\Delta t$ is taken as 5 seconds. With these numbers, the numerical horizontal momentum diffusivities vary from $8 \times 10^{2} \mathrm{~m}^{2} / \mathrm{s}$ to $2 \times 10^{4} \mathrm{~m}^{2} / \mathrm{s}$, which are well within the ranges intended to represent the physical horizontal momentum diffusivity in the oceans (e.g, Brian et al 1975, Bryan 1986).

If the horizontal momentum diffusivities can be well estimated, one may use the following formula to calculate $\beta_{\mathrm{u}}$ and $\beta_{\mathrm{v}}$

$$
\left(\beta_{\mathrm{u}}, \beta_{\mathrm{v}}\right)=4 \Delta \mathrm{t}\left(\frac{\mathrm{Ah}_{\mathrm{u}}}{\Delta \mathrm{x}^{2}}, \frac{\mathrm{Ah}_{\mathrm{v}}}{\Delta \mathrm{y}^{2}}\right)
$$

where $\mathrm{Ah}_{u}$ and $\mathrm{Ah}_{v}$ represent the horizontal momentum diffusivities, which are usually taken to be equal in the oceans.

\subsection{Combination of explicit and implicit schemes for stability}

With the time derivatives approximated as in the Eqs. (45) to (47), Eq. (37) can now be discretized in time

$$
\left[\begin{array}{l}
\eta_{1}^{(\tau+1)} \\
\mathrm{U} \\
\mathrm{V}
\end{array}\right]^{(\tau+}-\left[\begin{array}{c}
\mathbb{I}_{\mathrm{e}} \eta \\
\mathbb{I}_{\mathrm{u}} \mathrm{U} \\
\mathbb{I}_{\mathrm{v}} \mathrm{V}
\end{array}\right]^{(\tau)}=\Delta t\left[\begin{array}{ccc}
0 & -\mathrm{D}_{x} & -\mathrm{D}_{y} \\
-\mathrm{G}_{x} & -\mathbb{O}_{u}-\mathrm{F}_{\mathrm{u}} & \mathrm{C}_{\mathrm{v}} \\
-\mathrm{G}_{y} & -\mathrm{C}_{\mathrm{u}} & -\mathbb{O}_{v}-\mathrm{F}_{\mathrm{v}}
\end{array}\right]\left[\begin{array}{l}
\eta^{\eta} \\
\mathrm{U} \\
\mathrm{V}
\end{array}\right]^{(\tilde{\tau})}
$$

where $\tilde{\tau}$ indicate a time step yet to be determined. If $\tilde{\tau}=\tau$, the above scheme is explicit. If let $\tilde{\tau}=\tau+1$, the scheme is implicit. An implicit scheme is usually good for the computational stability, but is expensive because the equations have to be solved simultaneously. An explicit scheme is economic in computation but it risks the computational stability. To balance between the computational cost and stability, the following combination of $\tilde{\tau}=\tau$ and $\tilde{\tau}=\tau+1$ becomes a reasonable choice, 


$$
\left[\begin{array}{l}
\eta_{1} \\
U \\
V
\end{array}\right]^{(\tau+1)}-\left[\begin{array}{c}
\mathbb{I}_{e} \eta \\
\mathbb{I}_{u} U \\
\mathbb{I}_{v} V
\end{array}\right]^{(\tau)}=\Delta t\left[\begin{array}{ccr}
0 & -D_{x} & -D_{y} \\
0 & -\mathbb{O}_{u} & C_{v} \\
0 & 0 & -\mathbb{O}_{v}
\end{array}\right]\left[\begin{array}{c}
\eta^{(\tau)} \\
U \\
V
\end{array}\right]^{(\tau)}+\Delta t\left[\begin{array}{ccc}
0 & 0 & 0 \\
-G_{x} & -F_{u} & 0 \\
-G_{y} & -C_{u} & -F_{v}
\end{array}\right]\left[\begin{array}{l}
\eta_{1}^{(\tau+1)} \\
U \\
V
\end{array}\right]^{(\tau)}
$$

which split the coefficient matrix into two matrices. The splitting is based on the consideration that the matrix associated with $\tau+1$ time step can be economically inverted. Eq. (53) can be rearranged as

$$
\left[\begin{array}{ccc}
\mathrm{I}_{\mathrm{e}} & 0 & 0 \\
\Delta \mathrm{tG}_{\mathrm{x}} & \mathrm{E}_{\mathrm{u}} & 0 \\
\Delta \mathrm{t} \mathrm{G}_{\mathrm{y}} & \Delta \mathrm{tC}_{\mathrm{u}} & \mathrm{E}_{\mathrm{v}}
\end{array}\right]\left[\begin{array}{l}
\eta_{\mathrm{U}} \\
\mathrm{U} \\
\mathrm{V}
\end{array}\right]^{(\tau+1)}=\left[\begin{array}{ccc}
\mathbb{I}_{\mathrm{e}} & -\Delta \mathrm{tD}_{\mathrm{x}} & -\Delta \mathrm{tD}_{\mathrm{y}} \\
0 & \mathbb{I}_{\mathrm{u}}-\Delta \mathrm{t} \mathbb{O}_{\mathrm{u}} & \Delta \mathrm{tC}_{\mathrm{v}} \\
0 & 0 & \mathbb{I}_{\mathrm{v}}-\Delta \mathrm{t} \mathbb{O}_{\mathrm{v}}
\end{array}\right]\left[\begin{array}{l}
\eta_{\mathrm{W}}^{(\tau)} \\
\mathrm{U} \\
\mathrm{V}
\end{array}\right]^{(\tau)}
$$

where $\mathrm{E}_{\mathrm{u}}=\mathrm{I}_{\mathrm{u}}+\Delta \mathrm{tF}_{\mathrm{u}}$ and $\mathrm{E}_{v}=\mathrm{I}_{\mathrm{v}}+\Delta \mathrm{tF}_{\mathrm{v}}$, which are diagonal. The inverse of the coefficient matrix on the LHS of the above equation can be easily found as

$$
\left[\begin{array}{ccc}
\mathrm{I}_{\mathrm{e}} & 0 & 0 \\
\Delta \mathrm{tG}_{x} & \mathrm{E}_{\mathrm{u}} & 0 \\
\Delta \mathrm{tG}_{y} & \Delta \mathrm{tC}_{\mathrm{u}} & \mathrm{E}_{\mathrm{v}}
\end{array}\right]^{-1}=\left[\begin{array}{ccc}
\mathrm{I}_{\mathrm{e}} & 0 & 0 \\
-\Delta \mathrm{E}_{\mathrm{u}}^{-1} \mathrm{G}_{x} & \mathrm{E}_{\mathrm{u}}^{-1} & 0 \\
-\Delta \mathrm{tE}_{\mathrm{v}}^{-1} \mathrm{G}_{y}+(\Delta \mathrm{t})^{2} \mathrm{E}_{\mathrm{v}}^{-1} \mathrm{C}_{\mathrm{u}} \mathrm{E}_{\mathrm{u}}^{-1} \mathrm{G}_{x} & -\Delta \mathrm{tE}_{\mathrm{v}}^{-1} \mathrm{C}_{\mathrm{u}} \mathrm{E}_{\mathrm{u}}^{-1} & \mathrm{E}_{\mathrm{v}}^{-1}
\end{array}\right]
$$

What have been actually inverted are the diagonal matrices $\mathrm{E}_{\mathrm{u}}$ and $\mathrm{E}_{\mathrm{v}}$. Their inversions are not expensive at all.

Eq. (54) can now be solved as

$$
\begin{aligned}
{\left[\begin{array}{l}
\eta \\
U \\
V
\end{array}\right]^{(\tau+1)}=\left[\begin{array}{lcc}
\mathbb{L}_{11} & 0 & 0 \\
\mathbb{L}_{21} & \mathbb{L}_{22} & 0 \\
\mathbb{L}_{31} & \mathbb{L}_{32} & \mathbb{L}_{33}
\end{array}\right]\left[\begin{array}{ccc}
\mathbb{W}_{11} & \mathbb{W}_{12} & \mathbb{W}_{13} \\
0 & \mathbb{W}_{22} & \mathbb{W}_{23} \\
0 & 0 & \mathbb{W}_{33}
\end{array}\right]\left[\begin{array}{l}
\eta \\
U \\
V
\end{array}\right]^{(\tau)} } \\
= \\
=\left[\begin{array}{lll}
\mathbb{L}_{11} \mathbb{W}_{11} & \mathbb{L}_{11} \mathbb{W}_{12} & \mathbb{L}_{11} \mathbb{W}_{13} \\
\mathbb{L}_{21} \mathbb{W}_{11} & \mathbb{L}_{21} \mathbb{W}_{12}+\mathbb{L}_{22} \mathbb{W}_{22} & \mathbb{L}_{21} \mathbb{W}_{13}+\mathbb{L}_{22} \mathbb{W}_{23} \\
\mathbb{L}_{31} \mathbb{W}_{11} & \mathbb{L}_{31} \mathbb{W}_{12}+\mathbb{L}_{32} \mathbb{W}_{22} & \mathbb{L}_{31} \mathbb{W}_{13}+\mathbb{L}_{32} \mathbb{W}_{23}+\mathbb{L}_{33} \mathbb{W}_{33}
\end{array}\right]\left[\begin{array}{l}
\eta \\
U \\
V
\end{array}\right]^{(\tau)}
\end{aligned}
$$

where $\mathbb{L}_{\mathrm{i}, \mathrm{j}}$ represents the sub-matrices of the lower triangular matrix given by the RHS of Eq. (55) and $\mathbb{W}_{\mathrm{i}, \mathrm{j}}$ represents the sub-matrices of the upper triangular coefficient matrix given by the RHS of Eq. (54).

If let $\mathrm{A}$ be the RHS of Eq. (57), we can have an updating equation in a compact matrix form as

$$
\left[\begin{array}{l}
\eta \\
U \\
V
\end{array}\right]^{(\tau+1)}=A\left[\begin{array}{l}
\eta \\
U \\
V
\end{array}\right]^{(\tau)}, \quad \tau=0,1,2, \cdots \tau_{\max }
$$

which is the target form as was aimed in Section 2.4 .

Heaps (1969) suggested the time step as $\Delta \mathrm{t}=\mathrm{r}[2 \min (\mathrm{R} \Delta \phi, \mathrm{R} \cos \phi \Delta \lambda) / \sqrt{\mathrm{gh}}]$ where $\mathrm{r}$ is a lower bound to bring the time step $\Delta t$ within the maximum value allowed by the CFL condition. For the India and Pacific domain, $r$ is set as 0.95 .

In the above, the explicit and implicit schemes have been combined into one, which was originally proposed by Sielecki (1968). In the absence of frictions, and without the LaxFriedrichs averaging, according to Sielecki, the scheme preserves the energy. When frictions or Lax-Friedrichs averaging is present, the energy decays with the time. 
Notice that in the above updating scheme, updating V can always "enjoy" the most recently updated $U$, but not the other way around. If this kind of asymmetry in updating $U$ and $V$ is undesirable, it can be remedied by developing a counterpart to Eq. (58) by following the same route from Eq. (53) to Eq. (58) but with the positions of $U$ and V exchanged. The two updating schemes can then be used alternatively in time. That is to say, if Eq. (58) is used from $\tau$ to $\tau+1$, then its counterpart should used from $\tau+1$ to $\tau+2$. In order to have one matrix equation, the two updating schemes should be further combined into one by multiplying the two updating matrices. Before the multiplication, the counterpart matrix has to be permutated to restore the same order as of $[\eta \mathrm{UV}]^{\mathrm{T}}$. The matrix multiplication will result in a two-step updating scheme, covering from $\tau$ to $\tau+2$.

The author of this paper tried both asymmetric and symmetric schemes, but did not find noticeable difference in the solutions with the India-Pacific grid. Therefore, only the asymmetric scheme, i.e. Eq. (57), is used in the simulations, of which the results are going to be presented in the rest of this chapter. However it is worthwhile to note here on how we can derive a symmetric updating in matrix form, in case we will need it in some other modelling settings.

\section{All-source Green's functions}

Having derived the LSWE in matrix form, we are now ready to define the ASGF and to give its algorithm. We can then compare the ASGF and the SSGF side by side in terms of their computational costs and their scopes of applications. An important part of the ASGF is the part that operates on the initial sea level distribution. This part will be called the amplitude weights. Visualization of the amplitude weights brings up a classical concept in wave dynamics to the surface: the domain of dependence. The boundary of the domain of dependence serves a co-time line. The trace left by the ever growing boundary is a map of tsunami arrival times at a POI. Recordings of the history of the amplitudes weights at a particular place link to the responses in sea level at a POI to the initial forcing exerted at that particular place.

\subsection{Definition and algorithm of the ASGF}

Eq. (58) can be equivalently expressed as

$$
\left[\begin{array}{l}
\eta \\
U \\
V
\end{array}\right]^{(\tau)}=A^{\tau}\left[\begin{array}{l}
\eta^{(0)} \\
U \\
V
\end{array}\right]^{(0)}, \quad \tau=1,2,3, \cdots \tau_{\max }
$$

where $A^{\tau}$ represent the $\tau$ th power of the matrix A. However the two equations differ dramatically in their computational costs. If the solutions at all the model grid points are required, Eq. (58) should be the choice for computational efficiency, since each time step only involves a matrix-vector multiplication. In this case, to choose (59) would be unwise, since, when $\tau \geq 2$, it would require the calculations of all the powers of the matrix $A$, which would be very expensive or infeasible when the size of the matrix is large. However, if only solutions at a few model grid points are interested, Eq. (59) provides a distinct advantage as shown below.

Assume that there is only one POI, say the $k t h \eta$-point, where the sea surface elevation is interested. According to Eq. (59), the time series of the elevation at the kth $\eta$-point can be written as 


$$
\eta_{\mathrm{k}}^{(\tau)}=\mathrm{A}^{\tau}(\mathrm{k},:)\left[\begin{array}{l}
\eta^{(0)} \\
\mathrm{U} \\
\mathrm{V}
\end{array}\right]^{(0)}, \quad \tau=1,2,3, \cdots \tau_{\max }
$$

The crux here is how to compute the matrix power $A^{\tau}(k,:)$ economically for a series of $\tau^{\prime}$ s. If we first calculate the power of the whole matrix, and then extracts the $k t h$ row from the result, the calculation would be too expensive. Can we obtain the value of just the kth row of the matrix power without calculations for the other rows? Once the question is asked like this, the answer follows naturally. The following simple algorithm allows us to achieve what we want:

$$
\begin{aligned}
\mathrm{G}^{(0)} & =\mathrm{I}(\mathrm{k},:) \\
\mathrm{G}^{(1)} & =\mathrm{G}^{(0)} \mathrm{A} \\
\mathrm{G}^{(2)} & =\mathrm{G}^{(1)} \mathrm{A} \\
& \vdots \\
\mathrm{G}^{(\tau)} & =\mathrm{G}^{(\tau-1)} \mathrm{A} \\
& \vdots \\
\mathrm{G}^{\left(\tau_{\max }\right)} & =\mathrm{G}^{\left(\tau_{\max }-1\right)} \mathrm{A}
\end{aligned}
$$

Each of the series $G^{(\tau)},(\tau=0,1,2, \cdots)$, is a row vector. The initial one, $G^{(0)}$, is a row vector of the identity matrix. $\mathrm{G}^{(1)}$ is simply a copy of the $k$ th row of the matrix $A$. $\mathrm{G}^{(2)}$ is the $k t h$ row of the second power of the matrix A. Eq. (63) avoids the calculation of $A^{2}=A \times A$, it only calculates the second power for the needed row. The multiplication of the vector-matrix iterates to produces higher orders of the matrix powers for the needed row only. Since the identity matrix can be viewed as the zeroth power of the matrix A, and the matrix A can be viewed as the first power of itself, the series $G^{(\tau)},(\tau=0,1,2, \cdots)$, can be uniformly referred as the $k t h$ row of the $\tau^{\prime}$ th power of the matrix A.

The series $G^{(\tau)},(\tau=0,1,2, \cdots)$ define the ASGF, evaluated at $t=\tau \Delta t(\tau=0,1,2, \cdots)$. Each $G^{(\tau)}$ has $\mathrm{N}$ columns, with $\mathrm{N}$ being the number of total grid points where $\eta-, \mathrm{U}$ - or $\mathrm{V}$-variables are defined. A column can be viewed as an information channel. Having $\mathrm{N}$ channels means that signals from $\mathrm{N}$ different sources can be simultaneously received and stored separately by a receiver. The superposition principle will allow the $\mathrm{N}$-channel signals to be linearly combined to quickly yield a full solution when a real-time event happens.

We can now form a new matrix $\mathrm{G}$ as a collector of the individual row vectors, i.e.

$$
G=\left[G^{(0)} ; G^{(1)} ; \cdots ; G^{\left(\tau_{\max }\right)}\right]
$$

where the semicolon inside the square bracket indicate the end of a previous row and the beginning of the next. A simple matrix-vector multiplication as

$$
\eta_{k}(\tau)=G \times\left[\begin{array}{l}
\eta^{\prime} \\
U \\
V
\end{array}\right]^{(0)}
$$

can then provide the solution times series at the POI in response to an initial setup anywhere in the model domain. The collector $\mathrm{G}$ is also called the ASGF.

In the above, $\eta_{\mathrm{k}}(\tau)$ represents the time series of the elevations at the $k t h \eta$-node. Note that the parenthesized $\tau$ here is not in a superscript position. A parenthesized $\tau$ in a normal 
position means a vector of time indexes, $\tau=\left[\begin{array}{llll}1 & 2 & \cdots & \tau_{\max }\end{array}\right]$. A parenthesized $\tau$ is in a superscript position means one of the possible time steps, i.e., $\tau=1,2, \cdots, \tau_{\max }$. A nonparenthesized $\tau$ in a superscript position means the exponent of the power.

For a different initial setup, corresponding to a new tsunami, one can simply substitute a new column vector for $\left[\eta^{(0)} U^{(0)} V^{(0)}\right]^{T}$, without modifying the matrix $G$. That is, once $G$ has been calculated, it can be repeatedly used. The evolution from any specified initial state is easily calculated from a simple matrix-vector multiplication. The determination of the matrix G may require significant computer resources, but the matrix-vector multiplication can be quickly performed even on an ordinary personal computer or on a web server.

In the above discussions, the k-index was taken to indicate a single POI. This was just for simplicity of presentation; nothing prevents use of the k-index to represent several distinct POIs. One can thus use the same algorithm to calculate ASGFs for several POIs simultaneously. Also, the algorithm is completely ready for parallel computations. When there are many POIs, we can assign different values of the k-indexes to different processors or even different computational platforms without any need for exchange of information between processors or computational platforms. It should be feasible to calculate and store ASGFs for worldwide important cities as POIs and the entire global ocean as the potential sources.

\subsection{Comparison with the single-source Green's functions}

It is convenient at this stage to make a comparison between the ASGF and the SSGF. The procedure to calculate an SSGF can be recaptured essentially as follows:

$$
\begin{aligned}
\mathrm{F}^{(0)} & =\mathrm{I}(:, \mathrm{k}) \\
\mathrm{F}^{(1)} & =\mathrm{AF}^{(0)} \\
\mathrm{F}^{(2)} & =\mathrm{AF}^{(1)} \\
& \vdots \\
\mathrm{F}^{(\tau)} & =\mathrm{AF}^{(\tau-1)} \\
& \vdots \\
\mathrm{F}^{\left(\tau_{\max }\right)} & =\mathrm{AF}^{\left(\tau_{\max }-1\right)}
\end{aligned}
$$

where $\mathrm{F}^{(\tau)}(\tau=1,2,3, \cdots)$ defines the SSGF.

Comparing the algorithm listed above and the one listed in Eqs. (61) to (65), we can contrast the differences the ASGF and the SSGF side by side as below:

\begin{tabular}{|l|ll|}
\hline$-\quad \begin{array}{l}\text { The ASGF starts with a row of the } \\
\text { dynamic matrix A. The row } \\
\text { corresponds to a receiver point. }\end{array}$ & $\bullet \begin{array}{l}\text { The SSGF starts with a column of the } \\
\text { dynamic matrix A. The column } \\
\text { corresponds to a source point. }\end{array}$ \\
\hline $\begin{array}{l}\text { The ASGF grows in time when a } \\
\text { vector-matrix multiplication iterates. }\end{array}$ & $\bullet \begin{array}{l}\text { The SSGF grows in time when a matrix- } \\
\text { vector multiplication iterates. }\end{array}$ \\
\hline $\begin{array}{l}\text { The ASGF concentrates the information } \\
\text { from N-sources on one receiver }\end{array}$ & $\bullet \begin{array}{l}\text { The SSGF spreads information from one } \\
\text { source over N receivers. }\end{array}$ \\
\hline
\end{tabular}

A full name for the ASGF may read as all-source-one-receiver Green's function; a full name of the SSGF may read as single-source-all-receiver Green's function. It should be clear now that the ASGF and the SSGF cost the same computationally, but differ dramatically in terms of the hazard preparedness: the ASGF prepares a single POI against the entire ocean as potential hazard sources; the SSGF prepares the entire ocean against a single potential 
source. Obviously we only need to care a place where we live, not the entire ocean, against potential hazards. Since we cannot know where a future hazard source point will be, we had better assume that any parts of the ocean can be the potential source points.

\subsection{Amplitude weights and domain of dependence}

The matrix G, as defined by Eq. (66), can be split into three groups of columns, according to their dynamic roles, as follows:

$$
\mathrm{G}=\left[\begin{array}{lll}
\mathrm{W} & \mathrm{M}_{\mathrm{u}} & \mathrm{M}_{\mathrm{v}}
\end{array}\right]
$$

Accordingly, Eq. (67) can be re-expressed as

$$
\eta_{k}(\tau)=\left[\begin{array}{lll}
W & M_{u} & M_{v}
\end{array}\right]\left[\begin{array}{l}
\eta_{U} \\
U \\
V
\end{array}\right]^{(0)}
$$

The above expression says that the elevations at a POI is weighted averages of the initial sea level distribution, $\eta^{(0)}$, plus mass fluxes due to the initial stream component vectors, $U^{(0)}$ and $\mathrm{V}^{(0)}$. The matrices of $\mathrm{M}_{\mathrm{u}}$ and $\mathrm{M}_{v}$ capture the compound effects of many cycles of mass divergence $\rightarrow$ sea surface gradients $\rightarrow$ mass divergence during the propagations of waves from source points all the way to a destination point.

If the initial stream components are zero, Eq. (70) can be reduced to

$$
\eta_{\mathrm{k}}(\tau)=W \eta^{(0)}
$$

This equation says that the matrix $\mathrm{W}$ transmits a tsunami wave from its source region to the sea levels at a destination. The elements in columns of $W$ specify the weights exerted by the individual source points, and hence may be referred as amplitude weights. For a particular time $t=\tau \Delta t(\tau=0,1,2, \cdots), W(\tau,:)$ is a row vector. The initial one, $W(0,:)$, has only one nonzero element in the $k t h$ column, whose value is 1 . At the initial time, the only disturbance that can affect the $k t h$ point is the disturbance at the $k t h$ point itself. As $\tau$ progresses, the number of the non-zero elements of $W(\tau,:)$ increases, which means that any disturbance within the vicinity can affect the $k$ th point, and such vicinity grows with the time.

It is worth of pointing out that the amplitude weights are always summed up to 1 . This means that the initial unit weight is merely fractioned and redistributed by the subsequent operations, reflecting the law of mass conservation. It is also worth noting that while ordinary averaging weights are always positively valued between 0 and 1 , the amplitude weights are valued between -1 and 1 . A negative amplitude weight means that a positive/negative initial sea level at a source point results in a negative/positive sea level response at a receiver point.

Coloring the $\eta$-points according to the values of the amplitude weights depicts a domain of dependence, a key concept in the classical wave dynamics (e.g., Knobe, 2000): the elevation at the $k t h$ n-point at a particular time only depends on the distribution of the initial sea level within the domain. Any $\eta^{(0)}$ distributed outside of the domain has no effect on the elevation at that time. The three panels in Figure 5 show the amplitude weights at $t=2,4$ and 6 hours, where a DART buoy (51407) offshore Hawaii is taken for the POI, as marked by the crosses in purple in the panels.

The domain of dependence grows with time, as can be seen clearly in the figure. A tsunami triggered offshore California will not hit Hawaii in two hours and will for sure in 6 hours. Whether this tsunami will hit Hawaii in 4 hours will depend on how far offshore this tsunami is initially distributed. 

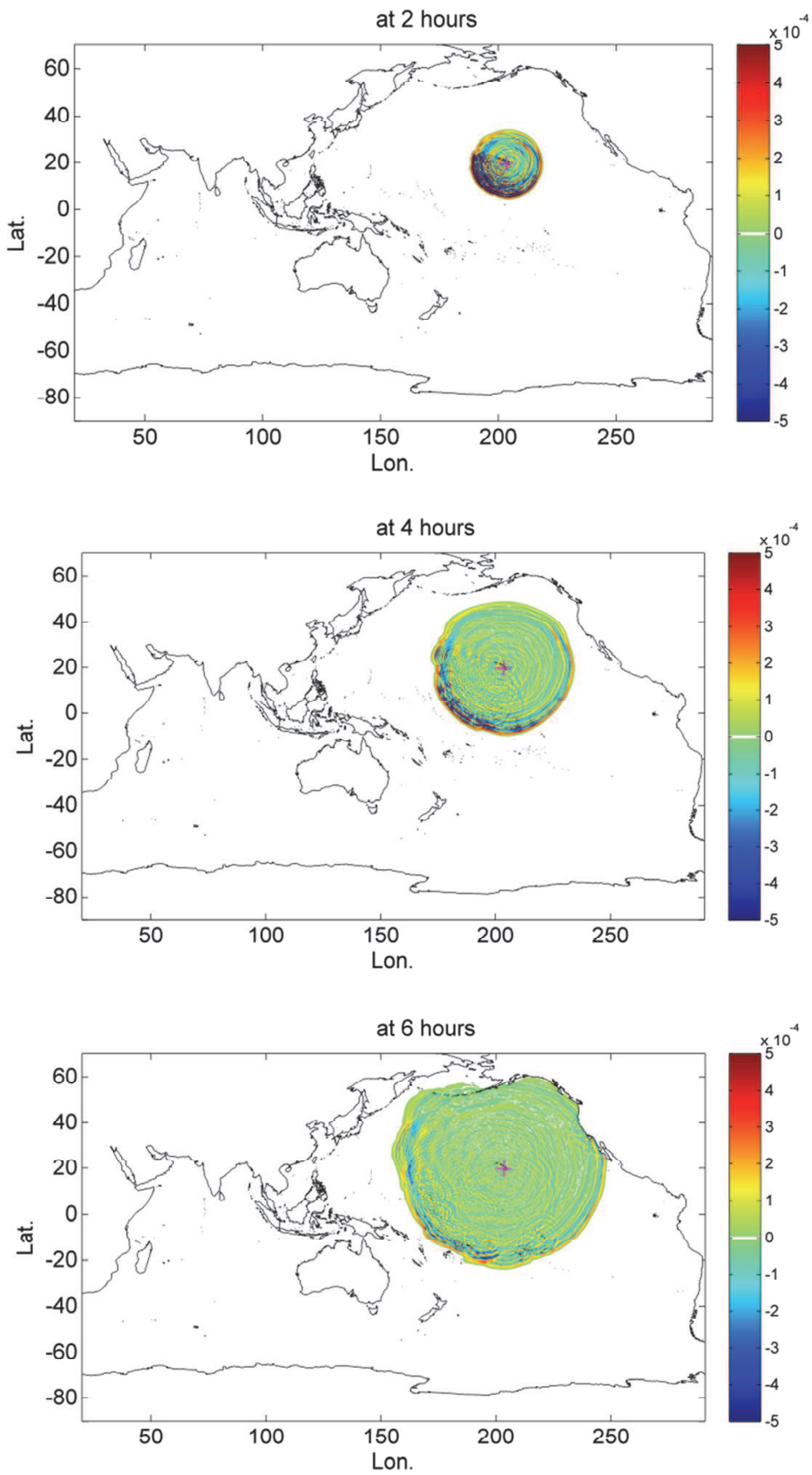

Fig. 5. Amplitude weights at 3 different times. The receiver point is marked by the crosses in purple, where a DART buoy (51407) is deployed. 
The boundary of the domain of dependence at a specific time can serve a co-time line. A disturbance on the boundary or within will arrive at the POI in time equal to or less than that specific time. Any disturbances outside the boundary have to take longer time to reach the target. A disturbance within a zone between the boundaries of domain of dependence corresponding to two specific times will arrive at the POI only between the two specific times.

\subsection{Response curves, Arrival Times (ATs), and Maximum Relative Amplitudes (MRAs)}

While a row of the matrix $W$ is a snapshot of the effects of all the cells at a particular time, a column of the matrix $\mathrm{W}$ contains the history of the effects by a particular cell. Shown in Figure 6 are three such histories of the effects on the same receiver by three distinct sources. In this example, the receiver is the DART station (51407) offshore Hawaii; the sources are three other DART stations $(21414,55015,56001)$. The duration of the time series is 36 hours. The vertical axes of the curves indicate the amplitudes relative to the source amplitudes. The green circles indicate the arrival times (ATs) of the first signal whose absolute value is above a threshold $\left(10^{-10}\right)$. The red circles indicate the values of the maximum relative amplitudes (MRAs) and their occurrence times as well. An MRA is the maximum absolute value seen in the curve for the entire duration. If the duration can be viewed long enough, an RMA then represents the maximum relative amplitude that can never be exceeded. From the curves, we can see that that the first waves are not always the largest.

The time step used in calculating the ASGFs is 5 seconds. Therefore one could store the results of the calculations as fine as 5 seconds, if this is needed. Or for the sake of disk space and the time needed for retrieving the data files, one may choose to save the results at a coarser interval such as 5 minutes, which is the case for the curves seen in Figure 6.

\section{Applications for tsunami preparedness and real-time simulations}

The time series as those in Figure 6 contain the details on the long wave dynamics. For a real-time simulation of the arrivals of an eventual tsunami, every bit of the information will be useful. In terms of tsunami preparedness, we only need to extract some pieces of information from the curves. The most important pieces of information we may read off are the ATs, the MRAs and MTs (maximum amplitude times). Each cell can be assigned with these values. Contouring and coloring the cells according to the assigned values give useful maps for tsunami preparedness.

\subsection{Arrival time map}

Figure 7 is an AT map for Hawaii. The destination point is marked by the white cross inside the 1-hour contour. This AT map tells when a tsunami will arrive at Hawaii should the tsunamis triggered somewhere in the India and Pacific Oceans. A traditional tsunami time map is a travel time map. It assumes a source point and contours the travel times to anywhere from the source point. However no one knows if a future tsunami will be indeed triggered at a pre-assumed source point. The arrival time map presented here focus on a point of destination instead, allowing source points to be everywhere.

\subsection{Maximum Relative Amplitude Map}

An MRA gives an upper bound on the relative wave amplitudes during a sufficient long time of a tsunami sourced at a particular point. Shown in Figure 8 is an MRA map, made for Hawaii. The map can be read as follows: 

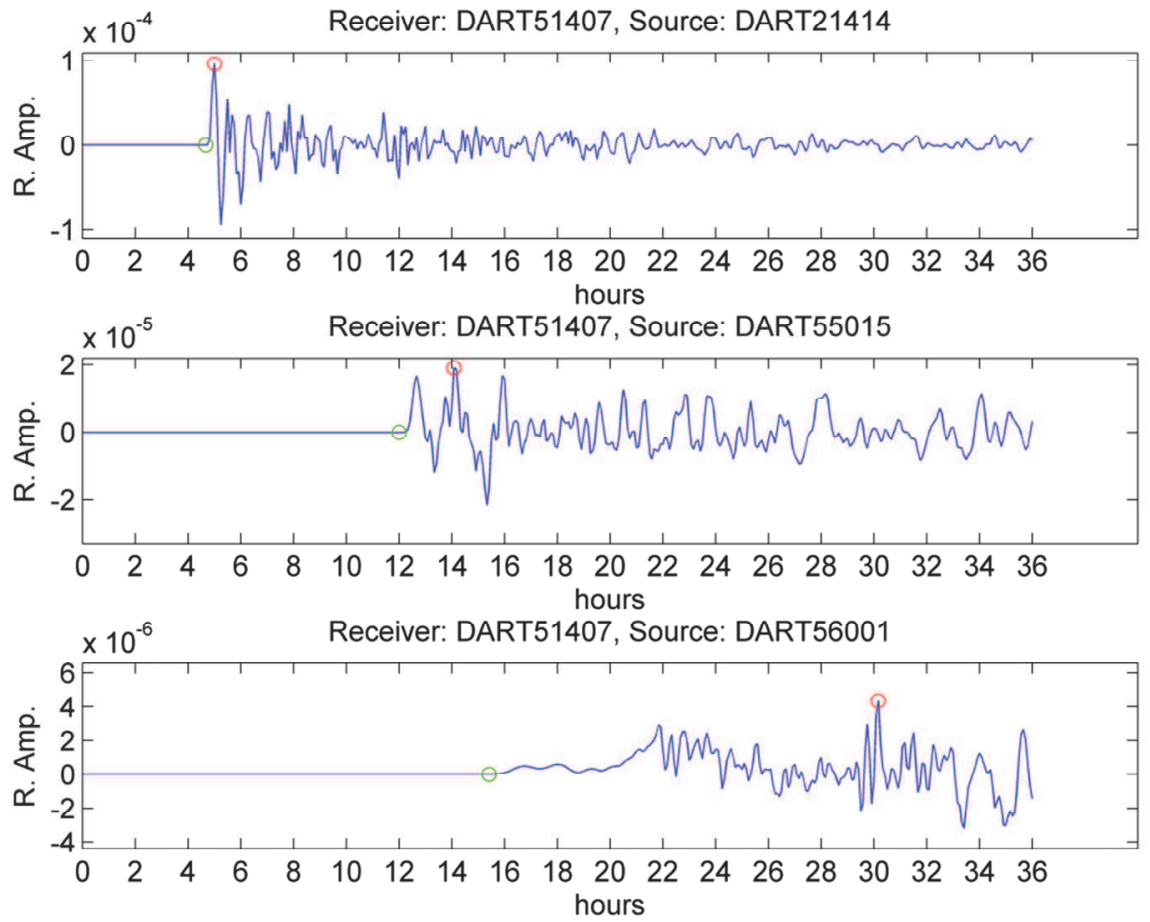

Fig. 6. Time series of relative amplitudes (R. Amp) at a receiver, DART 51407, as responses to the three impulse signals placed at three other DART stations $(21414,55015,56001)$.

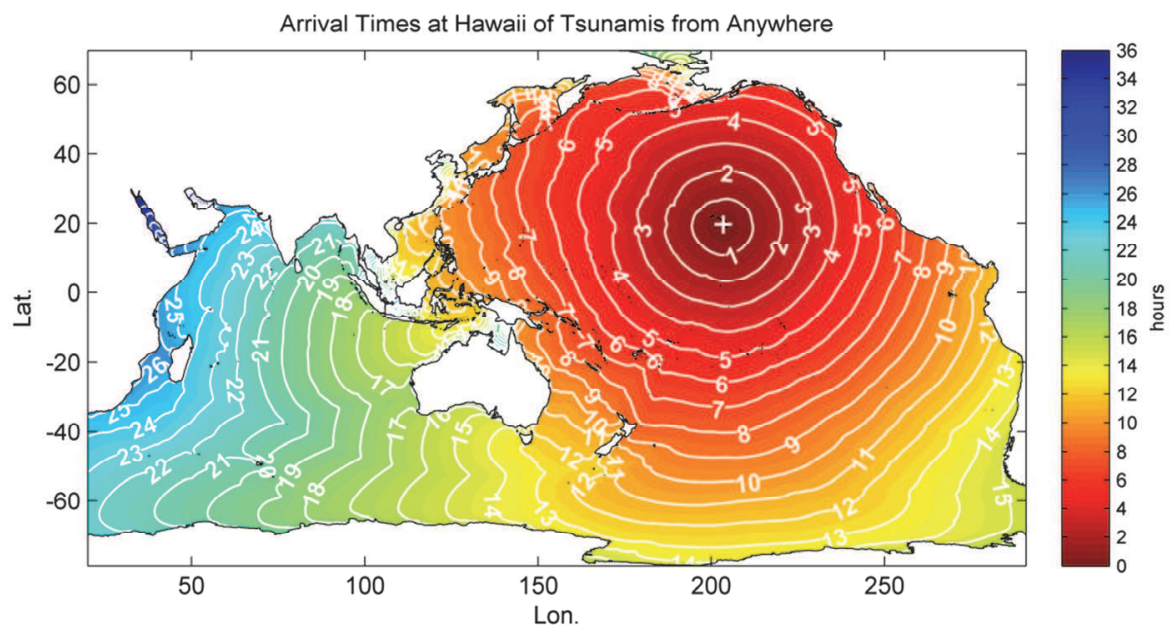

Fig. 7. A map of arrival times at Hawaii of tsunamis from anywhere in the India and Pacific Oceans. The destination point is marked by the white cross inside the 1-hour contour where the DART buoy 51407 is deployed. 


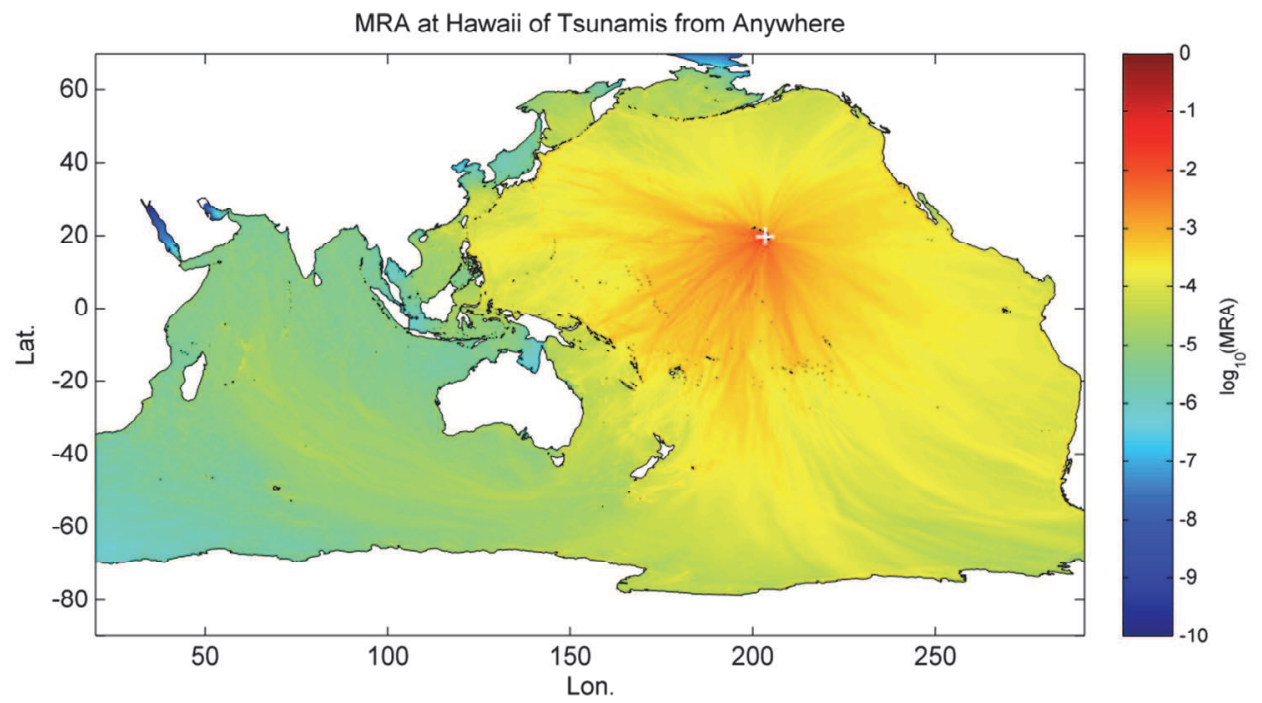

Fig. 8. Maximum relative amplitudes at Hawaii of tsunamis sourced anywhere in the India and Pacific Oceans. The destination point is marked by the white cross where the DART buoy 51407 is deployed.

Should someone be able to raise the mean sea level somewhere by one meter over the area occupied by a grid cell there and then suddenly release it, the dropping water column would generate waves propagating out in all directions. The waves would reach Hawaii eventually, and could be recorded by the DART buoy deployed there (please forget the sensitivity of the instrument in this thought experiment). As time goes by, the DART would yield a time series of the wave amplitudes. The largest amplitude one would see from the time series has already been predicted by the color on the map over the place where the water column was raised and dropped off.

The values of MRAs are all very small, ranging from 0 to 1 . This is because each of them represents the impact of a very small water column, whose height is $1 \mathrm{~m}$ and whose base area is the area of a cell, which is less than $10 \times 10 \mathrm{~km}^{2}$ on the grid with a 5-minute spacing. The potential energy stored in the water column has to spread out over the entire ocean. In order to see the small MRA values well, their $\log 10$ values are mapped to colors instead.

The values below $10^{-10}$ are not shown on the map. Any values below this threshold can be safely discarded for the grid in question. This is because that the total number of the grid cells is less than $4 \times 10^{6}$. If the maximum source amplitude was $10^{2} \mathrm{~m}$ and evenly distributed over a source region which occupies 1/100 of the entire India-Pacific Oceans (which is as large as $1 / 3$ of the area of Australia), the contribution by the discarded MRA part would only account for not more than $4 \times 10^{-4} \mathrm{~m}$ at the POI.

A tsunami triggered nearby a target has more impact than those triggered in a far field. The colors on the map visualize such differences in details. In contrast to the more or less cocircular contours seen in the arrival time map (Figure 7), the MRA map shows a strip pattern. This indicates that not all the points with the equal distances to a target have the same impact on the target. The variations in the water depths and the other factors such as 
wave reflections/refractions/interference all contribute to the differences. Knowledge on tsunami arrival times and knowledge on tsunami amplitudes are equally important. A time map and an amplitude map together offer us complementary information.

The concept of the maximum relative amplitude (MRA) was referred as a gain in Xu (2007). The term was coined because it had a sense of percentage of wave amplitude left when an initially unit amplitude wave arrived at a destination. Now the matrix form of the shallow water equations, as shown by Eq. (55), has rendered itself as a natural connecting point to the Kalman filter method. This is good for further studies on the assimilations of data coming from observatory stations such as DART buoys. In Kalman filter, there is an important concept called gain as well. In view of the potential name conflict, the term gain is replaced by the maximum relative amplitude, which is also more self-descriptive albeit it is longer.

\subsection{Maximum Relative Amplitude Time (MT) Map}

The occurrences of MRAs do not always immediately follow the arrivals of the initial signals, as illustrated by Figure 6. Therefore it is desirable to have also a map of the times when the MRAs occur. For brevity, let a map of this type called an MT map. Shown in Figure 9 is an MT map for Hawaii. In comparison with the AT map shown in Figure 7, the color bands in the MT time map are much less smooth even very rough in far-fields. This is because the MRAs are subjected to more complicated wave interactions. The initial arrival time is basically a kinematic issue. One could use the $\sqrt{g} h$ as the wave speed to make time contours and the results would be very close to what is shown in Figure 7. The time of the occurrence of an MRA is a dynamic issue. There is no simple rule as $\sqrt{g} h$ one could use to approximate the results as seen in Figure 9. In terms of time recording, the initial arrival times will never change once they are recorded. By contrast, maximum amplitude times are subjected to update if any subsequent waves exceed the previous ones.

It should be useful to know when a tsunami will initially arrive and when it will reach the maximum. Comparison of an AT map with an MT map gives us a bird's view of the difference between the two time points at a POI for tsunamis coming from anywhere in the oceans.

\subsection{Density of Maximum Relative Amplitudes}

The MRAs discussed above bear the influence of the grid cell sizes. Two water columns raised to the same height but over two cells of different sizes would have different impacts at the same destination point. If all the other affecting factors are the same on the their trips to the destination, the waves generated by the water column over the larger cell would impact more than the waves generated by the columns over the smaller cell. It is not necessary to pass on such cell size dependence to users of an MRA map, albeit the dependence may not be significant when the cells are fine.

To make an amplitude map free of the influences of cell sizes, we may normalize the MRAs by the cell areas. Let $\mathrm{E}$ be a diagonal matrix whose diagonal elements consist of the areas of all the water cells, we can transform Eq. (74) as

$$
\eta_{\mathrm{k}}(\tau)=\left(\mathrm{WE}^{-1}\right) \mathrm{E \eta}^{(0)}
$$

The largest values along the columns of $\mathrm{WE}^{-1}$ are the maximum relative amplitude per unit area, and may thus be referred as the densities of maximum relative amplitudes (DMRAs). 


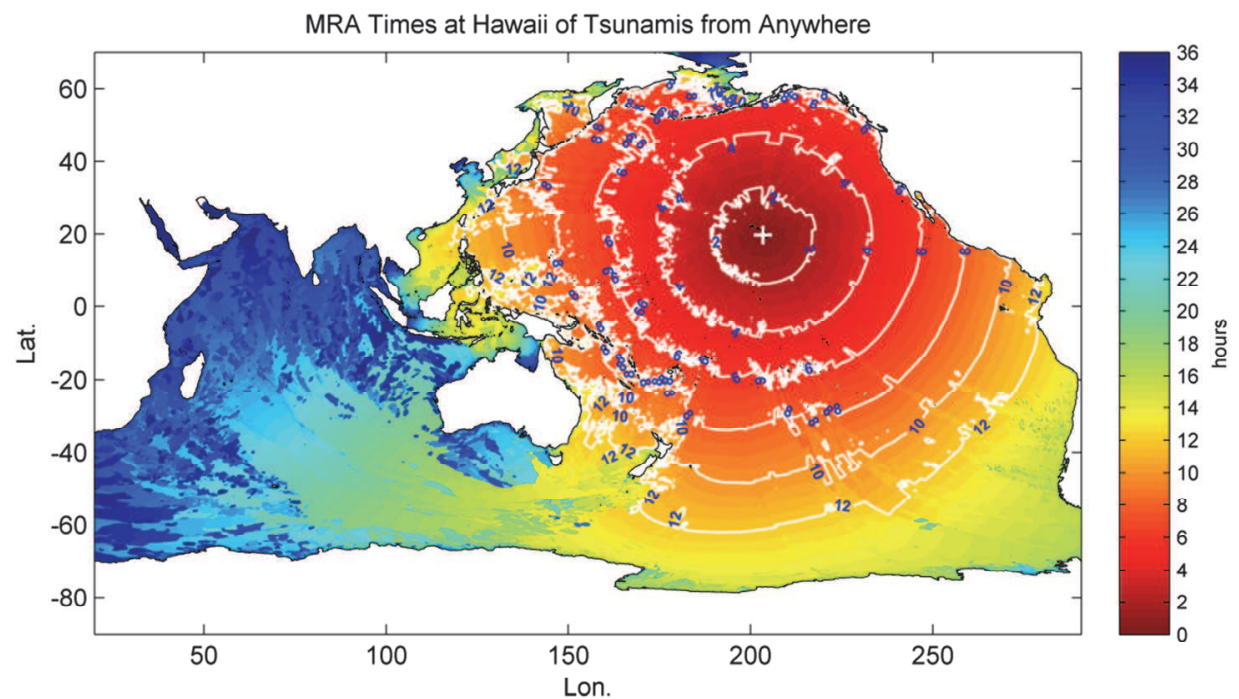

Fig. 9. A map of times when the maximum relative amplitude occur. For clarity, the contour lines are not superimposed as many as those in Figure 7.

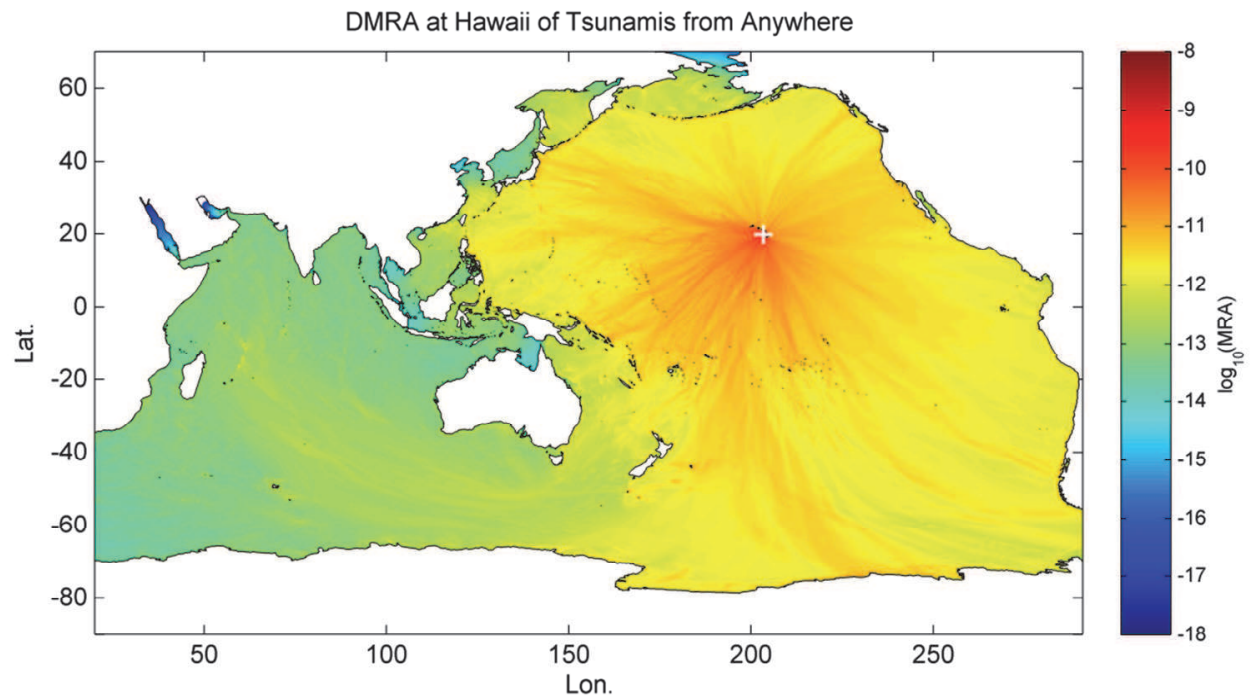

Fig. 10. A DMRA map showing the densities of maximum relative amplitudes.

A DMRA map can be read as follows: should someone be able to raise a $1 \mathrm{~m}$ water column over a 1- $m^{2}$ area somewhere on the map, the color on that spot indicates the largest amplitude that Hawaii would receive. The area-normalization makes the map area integrable, which means a user can integrate the DMRA over the area of potential hazard regions, and come up with an upper bound estimate of the largest relative amplitude or even better, the largest absolute amplitude if he can also estimate on the distribution of the wave amplitudes in the source region. 
Note that maps as presented above are not area preserving. The spherical surface areas at high latitudes (both south and north) are distorted on the paper. Therefore when a user exercises an area integration, he has to correct for the area distortion. To relieve users from this burden, the DMRA had better be presented on an area-preserving map. Figure 11 shows the same DMRAs but on a map made with the sinusoidal projection. Note that the units in $x$ and $y$ are all in meters on the map, and that the line of $x=0$ on the map corresponds to the $155^{\circ}$ longitudinal line.

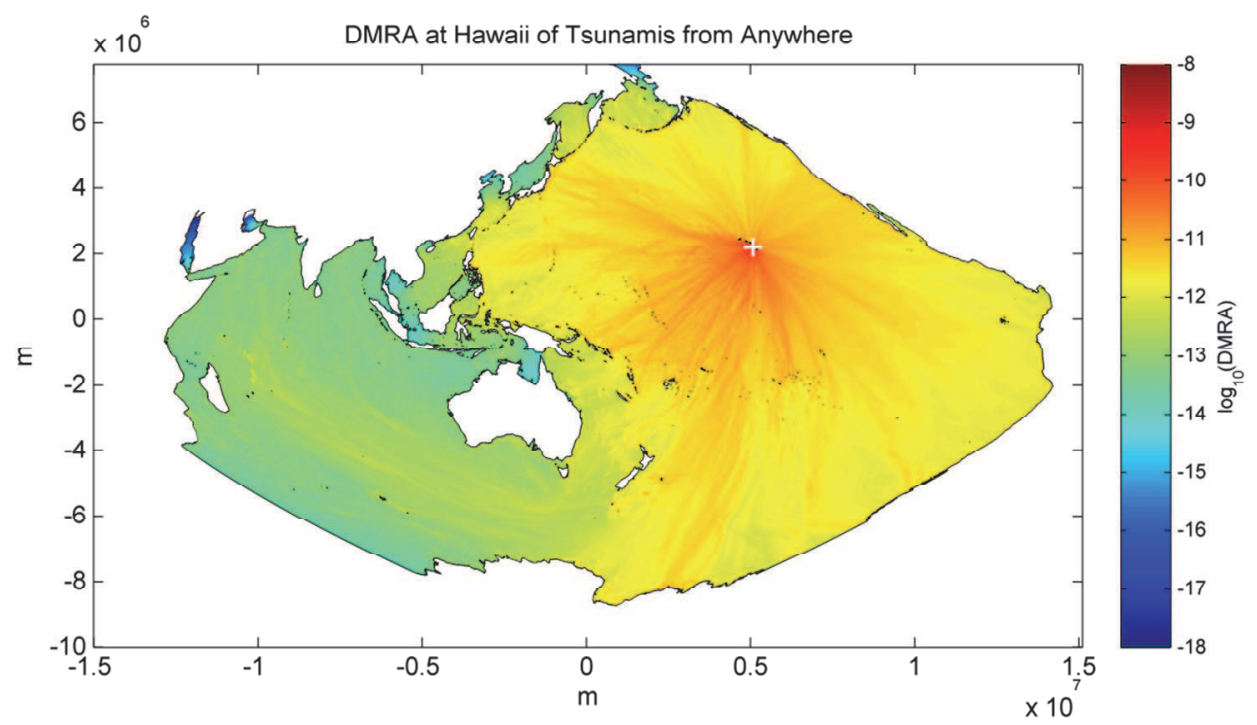

Fig. 11. Densities of maximum relative amplitudes shown an area-preserving map. The map results from using the sinusoidal projection. The line of $x=0$ corresponds to $155^{\circ}$ longitude line.

\subsection{Real-time simulations}

So far the amplitude weight matrix $\mathrm{W}$ has been used only in a summary manner: the important pieces of information are summarized into maps. With the complete dynamic information the weight matrix contains, we can go further: the real time simulations of tsunami arrivals from anywhere in the oceans.

To simulate tsunami propagations in real-time has been a great challenge. When a tsunami is triggered, it is very difficult to run a model on the fly in a vast transoceanic domain and yet still win the race for time against a fast moving tsunami $(\sim 700 \mathrm{~km} /$ hour $)$ even with a super computer and massively parallel computations. Now with Eq. (74), we can instantaneously transfer any disturbance at a source region to the sea level responses at a destination, no matter how far apart the source and the destination points are. This will give a valuable time window for tsunami warnings.

The weight matrix $\mathrm{W}$ in Eq. (74) has been pre-calculated and stored in a hard disk. If the matrix W has already been in the RAM of a computer, which may well be the case in an institute designated for tsunami warnings, it will take no time to compute just one matrixvector multiplication. If the weight matrix $W$ has not been in the RAM, it may take a few 
seconds to retriever it from the disk. Thus, with a tolerance of a few seconds, we can say that simulations of tsunami arrivals at a POI can always be performed instantaneously in realtime.

Based on Eq. (74), one can develop a desktop or web-based application for simulations of the arrivals at one or more POIs of tsunamis from anywhere in the oceans. Shown in Figure 12 is such a web-based application. The graphical user interface (GUI) shows a map with all the 40 DART stations as POIs. Forty weight matrices have been pre-calculated for a duration of 36 hours, and can be called for to deliver the responses upon receiving users' instructions through the interactive map.

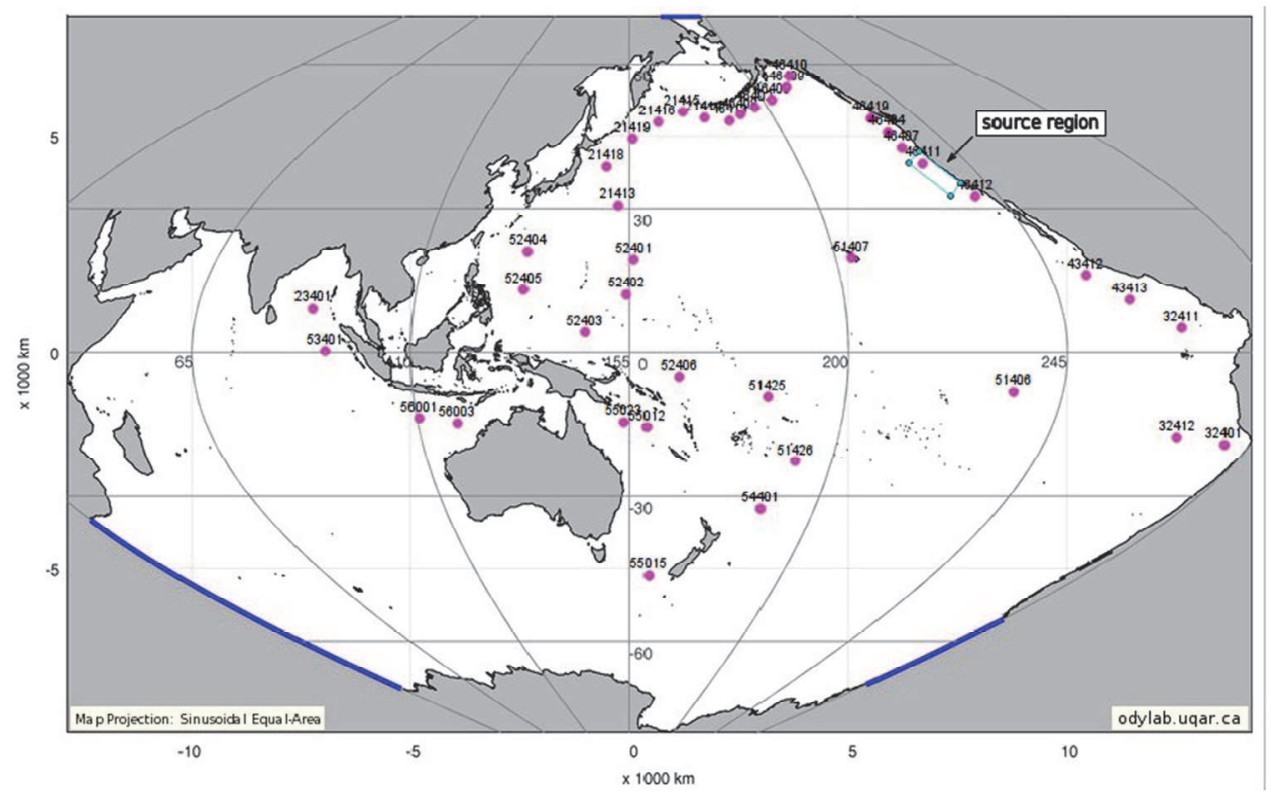

Choose among these points of interest:

\begin{tabular}{|c|c|c|c|c|c|c|}
\hline DART_21413 & DART_21414 $\square$ & DART_21415 & DART_21416 & DART_21418 & DART_21419 & $23401 \square$ \\
\hline ART_32401 & DART_32411 @ & DART_32412 D & DART_43412 & DART_43413 D & DART_46402 & DART_46403 \\
\hline ART_46404 & DART_46407 & DART_46408 & DART_46409 & DART_46410 & DART_46411 & DART_4 \\
\hline DART_46413 & DART_46419 & DART_51406 & DART_51407 $\checkmark$ & DART_51425 & DART_51426 & DART_52401 \\
\hline ART $52402 \square$ & DART 52403 & DART $52404 \square$ & DART $52405 \square$ & 52406 & DART 53401 & DART \\
\hline & 5015 & 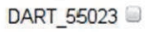 & DART_56001 & בח & All. & \\
\hline
\end{tabular}

Make plot!

See the plot(s) in the pop-up window.

04.44 sec

Fig. 12 A Real-time simulation system for tsunami arrivals at DART stations from anywhere in the India and Pacific Ocean. On the map, a source region offshore California is specified, and two POIs are selected (DART 55012 and 51407). It takes less than 5 seconds for the simulation system to deliver the response curves (see Figure 13). 

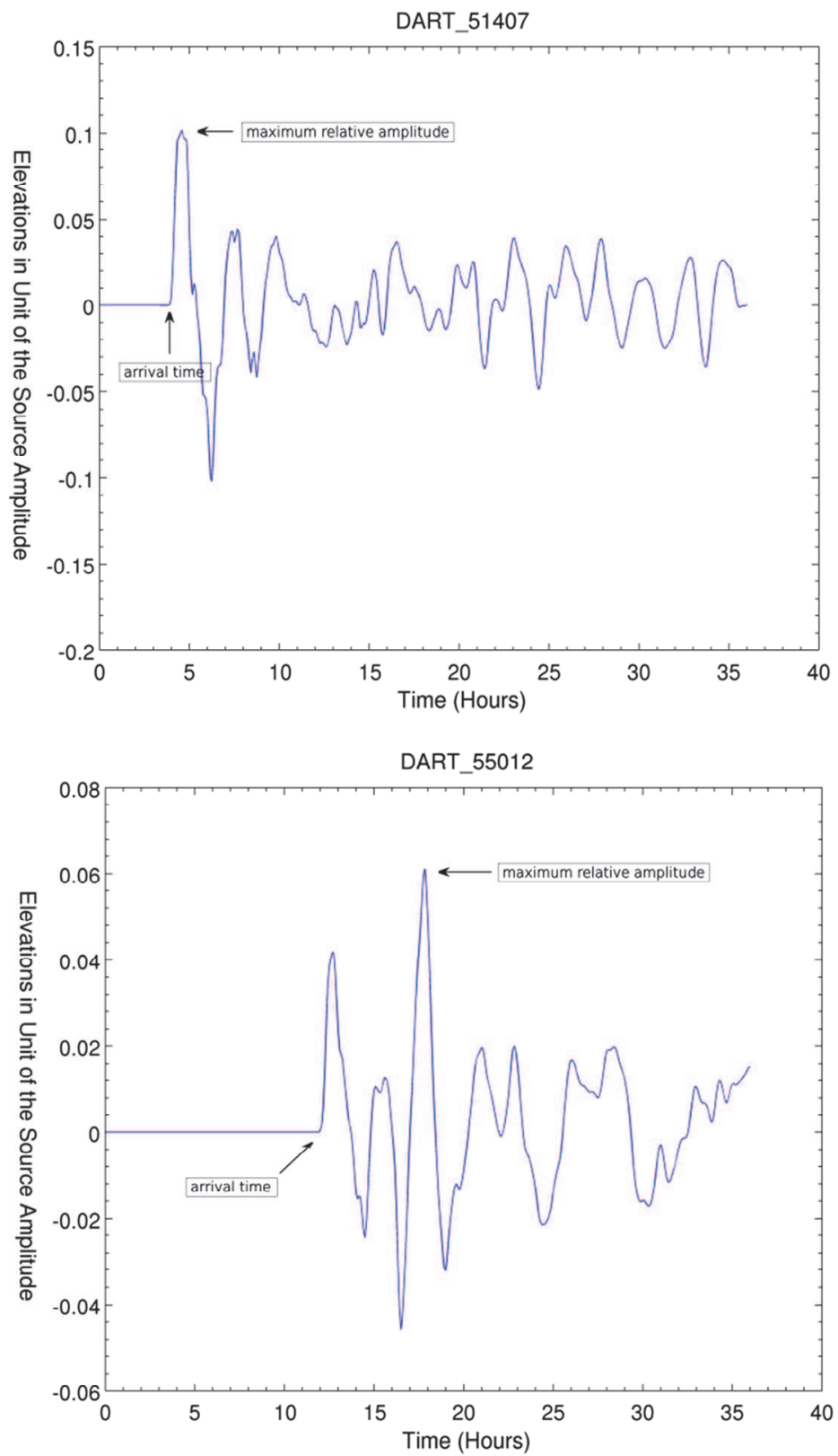

Fig. 13. Response curves by DART 51407 and DART 55012 to a tsunami source region offshore California (cf. Figure 12).

A user can arbitrarily click some points on the map to create a polygon defining a tsunami source region. Then he can choose one or more points of interest by checking the boxes in 
the list beneath the map. In a few seconds after pressing the "Make plot!" button on the lower left part of the GUI, he will see the tsunami arrival curves in a pop-up window. For the example shown in the figure, a rectangular source region was specified offshore California, and two POIs were chosen, DART 51407 and DART 55012. As we can see from the little timing box below the "Make plot" button, it took less than 5 seconds to deliver two response curves, which are shown in Figure 13.

For the above example, it was assumed that sea surface in the source region was uniformly raised by $1 \mathrm{~m}$ above the mean sea level. In other words, the sea level heights shown in Figure 13 are only relative the sea levels in the source region. If a realistic source distribution could be estimated or supplied by a tsunami genesis model, the units of response curves would then become absolute.

To reduce the time needed to retrieve them back from a hard disk, the amplitude weights should not be saved in a single large data file. Instead, they should be saved in many small data files. One may achieve this is by first tiling the model domain, then regrouping the columns of the weight matrix W such that each group of the columns corresponds to a tile. A group of the columns can then be saved as a small file. When a tsunami source region is specified, the tiles that the source region falls into can be detected, and hence only the data files belonging to those tiles need to be read off the disk. For a 5 minute grid spacing, a tile size of $10^{\circ} \times 10^{\circ}$ seems a good balance between the number and the sizes of the data files.

\section{Conclusions and future work}

A tsunami has three life phases: the genesis, the open-ocean propagation and the onshore runup. Towards a complete real time simulation system which covers the whole life of a tsunami, there are distinct challenges in each of the three phases to overcome. The challenges in the first and last phases lie in the dynamics complexity. Not all the earthquakes underneath the oceans can generate tsunamis. For simulations of onshore run-ups on the other end, the highly nonlinear tsunami bores over a complex onshore land and lack of timely and reliable open water boundary conditions are the conceivable challenges.

The challenges in the second phase are not due to dynamics complexity. Rather the dynamics in this phase is fortunately simple. The biggest challenge in this stage come from the fact that tsunamis run too fast in oceans that are too big for a propagation model to run on the fly and yet still be able to win a sufficient time window for tsunami warnings. The all-source Green's function (ASGF) offers a new approach to cope with this challenge. With a pre-calculated ASGF, simulations of tsunami arrivals become instantaneously realizable, no matter where and how far a tsunami comes from. As long as supplied with a reliable source function (the $\eta^{(0)}$ in Eq. (74)), a set of ASGFs can predict sea level anomalies for a set of points along a line offshore a coastal area, furnishing a local run-up model with necessary open water boundary conditions to simulate the onshore run-ups for that coastal area. The ASGF approach offers a good and fast link between the first and the third phases.

The web-based real time simulation system demonstrated in the chapter is just a starting point towards a complete system. Much more work can be done to improve it: linking with a genesis model and a run-up model on the two ends; linking with and assimilation of the live data from the DART buoys and other observational stations; important coastal cities from the Pacific Rim and countries along the India Ocean can be added as POIs in the system. The domain of the system can be increased too to include all the oceans on the planet.

For tsunami preparedness, the ASGF also brings in new types of tsunami maps: maps of tsunami arrival times, maps of maximum relative amplitudes and their occurrence times. 
These maps can also be helpful in a real-time when a tsunami is triggered. For example, whether a number read off an MRA map is above $70 \%$ or below $10 \%$ will make a big different to an emergence management officer in making his decision. An atlas of such maps can be made in the future for importance coastal cities around the world.

\section{Acknowledgement}

The author wishes to sincerely thank Dr. Dave Brickman for his careful review of the manuscript and valuable comments, Prof. Ted Murty for his very encouraging comments, and Mr. Michel Beaulieu for his help on the ODYLAB web page development. Thanks also go to Prof. Jarle Berntsen for the personal communications on the Coriolis weighting scheme.

\section{References}

Arakawa, A. \& Lamb, V. R. (1977). Computational design of the basic dynamical process of the UCLA general circulation model. Methods in Computational Phsics. 17, Academic Press, 173-265.

Bryan, K.; Manabe S. \& Pacanowski R. C. (1975). A global ocean-atmosphere climate model. Part II. The oceanic circulation, Journal of Physical Oceanography, 5, 30-46.

Bryan, F. 1987. Parameter sensitivity of primitive equation ocean general circulation models. J. Phys. Oceanogr., 17, 970985

Brummelhuis, P. G. J.; Heemink, A. W. \& Van Den Boogaard, H. F. P. (1993) Identification of shallow sea models. International journal for numerical methods in fluids. vol. 17, 637-665.

Chapman, D. C. (1985). Numerical Treatment of Cross-Shelf Open Boundaries in a Barotropic Coastal Ocean Model. Journal of Physical Oceanography, 15: 1060-1075

Ding, Y.; Jia Y.; ASCE, M.; Wang, S. S. Y. \& ASCE, F. (2004). Identification of Manning's Roughness Coefficients in Shallow Water Flows. Journal of Hydraulic Engineering, ASCE, June 2004.

Espelid, T. O; Berntsen J. \& Barthel K. (2000): Conservation of Energy for Schemes Applied to the Propagation of Shallow-Water Inertia-Gravity Waves in Regions with varying Depth. Int. J. Numer. Engng 2000; 49:1521-1545

Fujima, K. (2001). Long wave propagation on large roughness, ITS Proceedings. Session 7, No. 7-22. pp. 891-895.

Heaps, N. S. (1969). A Two-Dimensional Numerical Sea Model. Phil. Trans. R. Soc. Lond. A October 30, 1969265:93-137.

Heaps, N.S. (1971). On the numerical solution of the three-dimensional hydrodynamical equations for tides and storm surges, in: Mémoires de la Société Royale des Sciences de Liège. 6e Série, Tome II: pp. 143-180.

Knobe, R. (2000) An introduction to the mathematical theory of waves. American Mathematical Society; 1 edition (2000). ISBN-10: 0821820397.

Pedloskey, J. (1979) Geophysical Fluid Dynamics. Springer Verlag, 624 pp. ISBN: 3-540-90368-2.

Shuto, N. (1991). Numerical Simulation of Tsunamis --- Its Present and Near Future. Natural Hazards 4: 171-191.

Sielecki, A. (1968): An Energy-Conserving Difference Scheme for the storm surge equations Mon. Wea. Rev., 96, 150-156.

Sommerfeld, A. (1949). Partial Differential Equations in Physics. Academic Press, New York, New York, 1949.

$\mathrm{Xu}, \mathrm{Z}$. (2007). The all-source Green's function and its applications to tsunami problems. Science of Tsunami Hazards, 26(1), 59-69. 


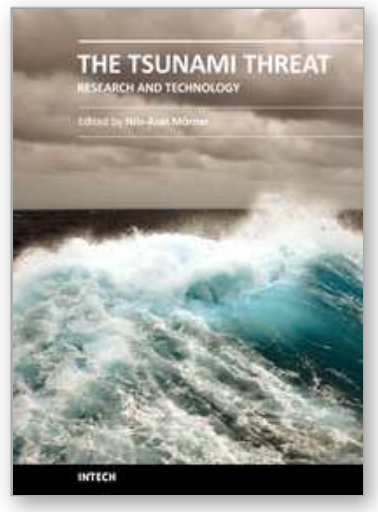

\author{
The Tsunami Threat - Research and Technology \\ Edited by Nils-Axel MÃ
}

ISBN 978-953-307-552-5

Hard cover, 714 pages

Publisher InTech

Published online 29, January, 2011

Published in print edition January, 2011

Submarine earthquakes, submarine slides and impacts may set large water volumes in motion characterized by very long wavelengths and a very high speed of lateral displacement, when reaching shallower water the wave breaks in over land - often with disastrous effects. This natural phenomenon is known as a tsunami event. By December 26, 2004, an event in the Indian Ocean, this word suddenly became known to the public. The effects were indeed disastrous and 227,898 people were killed. Tsunami events are a natural part of the Earth's geophysical system. There have been numerous events in the past and they will continue to be a threat to humanity; even more so today, when the coastal zone is occupied by so much more human activity and many more people. Therefore, tsunamis pose a very serious threat to humanity. The only way for us to face this threat is by increased knowledge so that we can meet future events by efficient warning systems and aid organizations. This book offers extensive and new information on tsunamis; their origin, history, effects, monitoring, hazards assessment and proposed handling with respect to precaution. Only through knowledge do we know how to behave in a wise manner. This book should be a well of tsunami knowledge for a long time, we hope.

\title{
How to reference
}

In order to correctly reference this scholarly work, feel free to copy and paste the following:

Zhigang Xu (2011). The All-Source Green's Function of Linear Shallow Water Dynamic System: Its Numerical Constructions and Applications to Tsunami Problems, The Tsunami Threat - Research and Technology, NilsAxel MÃ rner (Ed.), ISBN: 978-953-307-552-5, InTech, Available from: http://www.intechopen.com/books/thetsunami-threat-research-and-technology/the-all-source-green-s-function-of-linear-shallow-water-dynamicsystem-its-numerical-constructions-a

\section{INTECH}

open science | open minds

\author{
InTech Europe \\ University Campus STeP Ri \\ Slavka Krautzeka 83/A \\ 51000 Rijeka, Croatia \\ Phone: +385 (51) 770447 \\ Fax: +385 (51) 686166 \\ www.intechopen.com
}

\author{
InTech China \\ Unit 405, Office Block, Hotel Equatorial Shanghai \\ No.65, Yan An Road (West), Shanghai, 200040, China \\ 中国上海市延安西路65号上海国际贵都大饭店办公楼 405 单元 \\ Phone: +86-21-62489820 \\ Fax: +86-21-62489821
}


(C) 2011 The Author(s). Licensee IntechOpen. This chapter is distributed under the terms of the Creative Commons Attribution-NonCommercialShareAlike-3.0 License, which permits use, distribution and reproduction for non-commercial purposes, provided the original is properly cited and derivative works building on this content are distributed under the same license. 